\title{
The Sunrise Mission
}

\author{
P. Barthol • A. Gandorfer · S.K. Solanki • M. Schüssler • B. Chares • W. Curdt • \\ W. Deutsch · A. Feller · D. Germerott • B. Grauf • K. Heerlein · J. Hirzberger • \\ M. Kolleck • R. Meller • R. Müller • T.L. Riethmüller • G. Tomasch • M. Knölker • \\ B.W. Lites - G. Card · D. Elmore · J. Fox • A. Lecinski · P. Nelson · R. Summers • \\ A. Watt • V. Martínez Pillet • J.A. Bonet - W. Schmidt • T. Berkefeld • A.M. Title • \\ V. Domingo · J.L. Gasent Blesa · J.C. del Toro Iniesta • A. López Jiménez • \\ A. Álvarez-Herrero · L. Sabau-Graziati · C. Widani - P. Haberler · K. Härtel · \\ D. Kampf • T. Levin • I. Pérez Grande · A. Sanz-Andrés · E. Schmidt
}

Received: 8 June 2010 / Accepted: 23 October 2010 / Published online: 24 November 2010

(C) The Author(s) 2010. This article is published with open access at Springerlink.com

\begin{abstract}
The first science flight of the balloon-borne Sunrise telescope took place in June 2009 from ESRANGE (near Kiruna/Sweden) to Somerset Island in northern Canada. We describe the scientific aims and mission concept of the project and give an overview and a description of the various hardware components: the 1-m main telescope with its postfocus
\end{abstract}

P. Barthol $(\bowtie) \cdot$ A. Gandorfer · S.K. Solanki · M. Schüssler · B. Chares · W. Curdt · W. Deutsch · A. Feller · D. Germerott · B. Grauf · K. Heerlein · J. Hirzberger · M. Kolleck · R. Meller · R. Müller · T.L. Riethmüller · G. Tomasch

Max-Planck-Institut für Sonnensystemforschung, Max-Planck-Straße 2, 37191 Katlenburg-Lindau, Germany

e-mail: barthol@mps.mpg.de

M. Knölker · B.W. Lites · G. Card · D. Elmore · J. Fox · A. Lecinski · P. Nelson · R. Summers · A. Watt High Altitude Observatory, P.O. Box 3000, Boulder, CO 80301, USA

V. Martínez Pillet · J.A. Bonet

Instituto de Astrofísica de Canarias, C/Vía Láctea, s/n, 38205 La Laguna (Tenerife), Spain

W. Schmidt · T. Berkefeld

Kiepenheuer-Institut für Sonnenphysik, Schöneckstraße 6, 79104 Freiburg, Germany

A.M. Title

Lockheed Martin Solar and Astrophysics Laboratory, Bldg. 252, 3251 Hanover Street, Palo Alto, CA 94304, USA

V. Domingo · J.L. Gasent Blesa

Grupo de Astronomía y Ciencias del Espacio, Univ. de Valencia, 46980 Paterna, Valencia, Spain

J.C. del Toro Iniesta · A. López Jiménez

Instituto de Astrofísica de Andalucía (CSIC), Apdo. de Correos 3004, 18080 Granada, Spain

A. Álvarez-Herrero · L. Sabau-Graziati

Instituto Nacional de Técnica Aerospacial, 28850 Torrejón de Ardoz, Spain 
science instruments (the UV filter imager SuFI and the imaging vector magnetograph IMaX) and support instruments (image stabilizing and light distribution system ISLiD and correlating wavefront sensor CWS), the optomechanical support structure and the instrument mounting concept, the gondola structure and the power, pointing, and telemetry systems, and the general electronics architecture. We also explain the optimization of the structural and thermal design of the complete payload. The preparations for the science flight are described, including AIV and ground calibration of the instruments. The course of events during the science flight is outlined, up to the recovery activities. Finally, the in-flight performance of the instrumentation is discussed.

Keywords Instrumentation and data management · Integrated sun observations · Magnetic fields, photosphere

\section{Introduction}

\subsection{Solar Balloon Missions}

A number of projects has been devoted in the past to observing the Sun from balloonborne platforms in the stratosphere. In 1957 and 1959, project Stratoscope (Schwarzschild, 1959) obtained white-light images of granulation and sunspots with a 30-cm telescope (Danielson, 1961). A different instrument called Spektro-Stratoskop took several long sequences of high quality granulation pictures with an evacuated 30-cm telescope (Mehltretter, 1978). From 1966 to 1973, there were several flights of the Soviet Stratospheric Solar Station, which operated alternatively with main mirrors of $50 \mathrm{~cm}$ and $1 \mathrm{~m}$ diameter and took filtergrams in the visible range (e.g. Krat, Karpinsky, and Pravdjuk, 1972). Smaller telescopes $(5-10 \mathrm{~cm})$ with broad-band filters in the visible were flown by a Japanese group in the 1970s (Hirayama, 1978). Groups in France attempted UV imaging in the wavelength range 200-300 nm with a 20-cm telescope (Herse, 1979) and took spectra in the same wavelength range with telescopes of up to 30-cm aperture from the mid 1960s on (Lemaire and Samain, 1984; Lemaire and Blamont, 1967). More recently, flights of the Flare Genesis project in 1996 and 2000 provided magnetograms and Dopplergrams in the Ca I 6122.2 line using a 80-cm telescope (Rust et al., 1996; Bernasconi et al., 2000). The 30-cm telescope of the Solar Bolometric Imager (Bernasconi et al., 2004) provided maps of the solar disk in integrated light between 0.28 and $2.6 \mu \mathrm{m}$ during three flights between 2003 and 2007.

These projects demonstrated the advantages and the rich potential of balloon-borne solar observations at stratospheric heights:

i) negligible image degradation by atmospheric seeing

ii) access to the UV range down to about 200-nm wavelength

C. Widani · P. Haberler · K. Härtel · D. Kampf · T. Levin

Kayser-Threde GmbH, Wolfratshauser Straße 48, 81379 München, Germany

I. Pérez Grande · A. Sanz-Andrés

Universidad Politécnica de Madrid, IDR/UPM, Plaza Cardenal Cisneros 3, 28040 Madrid, Spain

E. Schmidt

Ingenieurbüro für Optikentwicklung, Amalienstraße 12, 85737 Ismaning, Germany 
iii) retrievability of the instruments, and

iv) much reduced cost in comparison to space projects

The Sunrise mission comprises the biggest and most complex payload flown so far in a solar balloon mission: a 1-m telescope equipped with a multi-wavelength UV filter imager, a Fabry - Pérot-based vector magnetograph, and a correlating wavefront sensor for active alignment control and image stabilization.

\subsection{Science Case}

The solar photosphere is crucial for the investigation of the solar magnetic field. This thin layer, where the plasma becomes optically thin and almost all of the radiative energy flux is emitted, represents the key interaction region: thermal, kinetic and magnetic energy all are of the same order of magnitude and transform most easily from one form into another. The interaction between convection, radiation, and magnetic field in the electrically conducting solar plasma leads to the creation of a rich variety of magnetic structure with intense (kilogauss) magnetic field concentrations on size scales reaching well below a pressure scale height. At the same time, the photosphere appears to harbor a stunning amount of mixedpolarity 'turbulent' magnetic flux, which possibly results from small-scale local dynamo action driven by the granulation flows. The photospheric magnetic field is in a state of constant change: ceaseless transport, stretching, emergence and submergence, cancellation, intensification and dissipation of magnetic flux take place down to the smallest spatial scales that can be observed so far. These processes control the structure, dynamics, and energetics of the solar atmosphere at larger scales; they are the source of solar variability and, ultimately, of solar influences on the Earth. To understand these fundamental processes, we must learn how the magnetic field interacts with the solar plasma and have to uncover the conversion of energy between its mechanical, magnetic, radiative and thermal forms. Consequently, the central questions of Sunrise science are the following.

- What are the origin and the properties of the intermittent magnetic structure, including the kilogauss concentrations?

- How is the magnetic flux brought to and removed from the solar surface? What is the role played by local dynamo action and reconnection processes?

- How does the magnetic field assimilate and provide energy to heat the upper solar atmosphere?

- How does the variable magnetic field modify the solar brightness?

In order to answer these questions, the key scientific objective of the Sunrise project is to study the structure and dynamics of the solar magnetic field at the spatial resolution afforded by a 1-m telescope, over extended stretches of time with constant observational conditions free of seeing effects, with a time resolution sufficient to track rapid changes of the magnetic field, and over a field of view large enough to provide good statistics of relevant events and to follow the evolution of magnetic structure during all phases of its life cycle.

The required observations include continuous, quantitative measurements of the magnetic field vector, the plasma velocity and related atmospheric structure, together with brightness maps in several wavelength bands. For the latter, the UV wavelengths between $200 \mathrm{~nm}$ and $300 \mathrm{~nm}$ are of particular interest since $i$ ) no high-resolution imaging has been done so far in this range and ii) cycle-related irradiance variations in this spectral band affect the temperature in the terrestrial stratosphere through ozone photochemistry. Numerical 
simulations predict intensity contrasts of magnetic flux concentrations at these wavelengths that significantly exceed those in the visible.

\subsection{Mission Concept}

Sunrise has been designed as a balloon-borne stratospheric solar observatory in the framework of NASA's LDB (Long Duration Balloon) program. Zero pressure helium balloons with volumes up to one million $\mathrm{m}^{3}$ lift science payloads such as Sunrise with a mass of several tons to float altitudes of $35-37 \mathrm{~km}$. This 'low cost access to near space' has several advantages compared to ground-based or space-borne solar instrumentation. As with spaceborne instruments, stratospheric solar observations do not suffer from two major drawbacks encountered with ground based telescopes. Being above $99 \%$ of the Earth's atmosphere, wavefront distortions due to atmospheric turbulence are virtually not existent. This provides long-term and seeing-free high resolution imaging conditions. In addition, the interesting solar UV radiation between $220 \mathrm{~nm}$ and $370 \mathrm{~nm}$ is accessible, which is absorbed in the lower stratosphere by the Hartley and Huggins bands of ozone. At mission termination balloonborne instruments usually can be recovered after landing with only moderate damage. Modifications for a future flight and refurbishment can be accomplished very cost effectively, at far lower investment levels compared to space instruments.

NASA LDB missions are managed and operated by the Columbia Scientific Ballooning Facility (CSBF), Palestine, Texas, USA and in the past have been launched mainly from Williams Field near McMurdo, Antarctica $\left(77.86^{\circ} \mathrm{S}, 167.13^{\circ} \mathrm{E}\right)$, with a launch window from December to January. NASA recently expanded their launch capabilities by cooperating with ESRANGE $\left(67.89^{\circ} \mathrm{N}, 21.10^{\circ} \mathrm{E}\right)$ near Kiruna, Sweden, allowing missions to be launched during the northern summer period as well. The European sounding rocket and balloon base provides excellent infrastructure and is accessible with much lower logistical effort compared to McMurdo. Launching balloons during solstice conditions close to the polar circle offers uninterrupted solar observations without day/night cycles. Permanent sunlight and only small elevation changes of the Sun form ideal conditions, so that undisturbed observation and power generation for the instruments are guaranteed. Furthermore, thermal conditions do not vary significantly and the balloon floats at nearly constant altitude. Relatively stable wind systems at float altitude take the balloon and instrument on circumpolar trajectories with flight durations of nine to 12 days per revolution. ESRANGE was chosen for the first science flight of Sunrise, although the flight duration currently is limited to five - six days due to the lack of Russian overflight permissions for NASA balloons. The processes to be studied by Sunrise generate highly variable structures like emerging and decaying active regions or sunspots. The timescales involved range from seconds on small spatial scales over minutes and hours to several days in case of sunspots. Due to their intrinsic nature these phenomena cannot be predicted in time and location. Being at the onset of the new solar activity cycle, the shorter flight duration was judged to be acceptable in comparison to the higher logistical effort and risk related with an Antarctic flight.

Coordinated measurements were done with Hinode and SUMER onboard SOHO. Parallel observations with ground-based solar telescopes such as the Swedish Solar Telescope (SST) on La Palma, the Vacuum Tower Telescope (VTT) on Tenerife, the IRSOL facility in Locarno (Switzerland), and the Dunn Solar Telescope on Sacramento Peak (New Mexico) have been performed to support the target selection for Sunrise. 


\section{Instrument Description}

Sunrise in full flight configuration is shown in Figure 1. The instrument can be broken down into the following major components, which will be described in more detail below:

- Telescope

- Postfocus instrumentation platform (PFI) with science instruments and image stabilization system

- Gondola with photovoltaic arrays and pointing system

- Instrument electronics

- CSBF-provided ballooning equipment

- Ground-support equipment

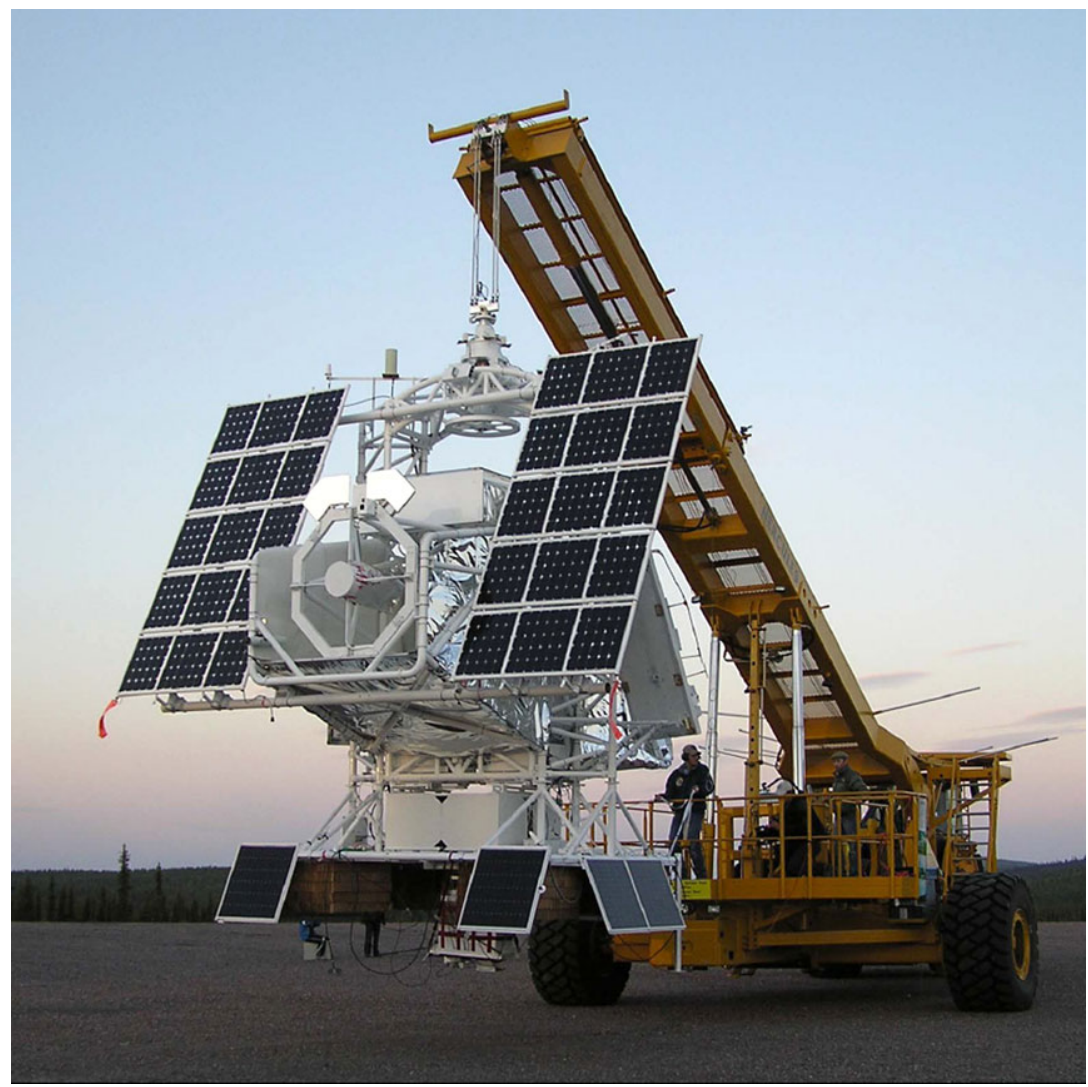

Figure 1 Overview of the Sunrise observatory in full flight configuration, immediately before launch. The dimensions of the observatory can be judged by comparing with the person on the launch vehicle. In the center of the gondola the telescope front ring can be seen, with the radiators for the heat rejecting prime focus field stop. The box on top of the telescope is the postfocus instrumentation (PFI) platform housing the science instruments. A flywheel below the upper bridge of the gondola structure is used for azimuth pointing. Instrument electronics is mounted on two inclined racks on the rear side of the gondola. The white box in the lower part of the gondola is the CSBF-provided system instrumentation package (SIP), needed for commanding of balloon and payload. It uses a dedicated power system, while the main power for the observatory is generated by the large solar arrays to both sides of the telescope. 


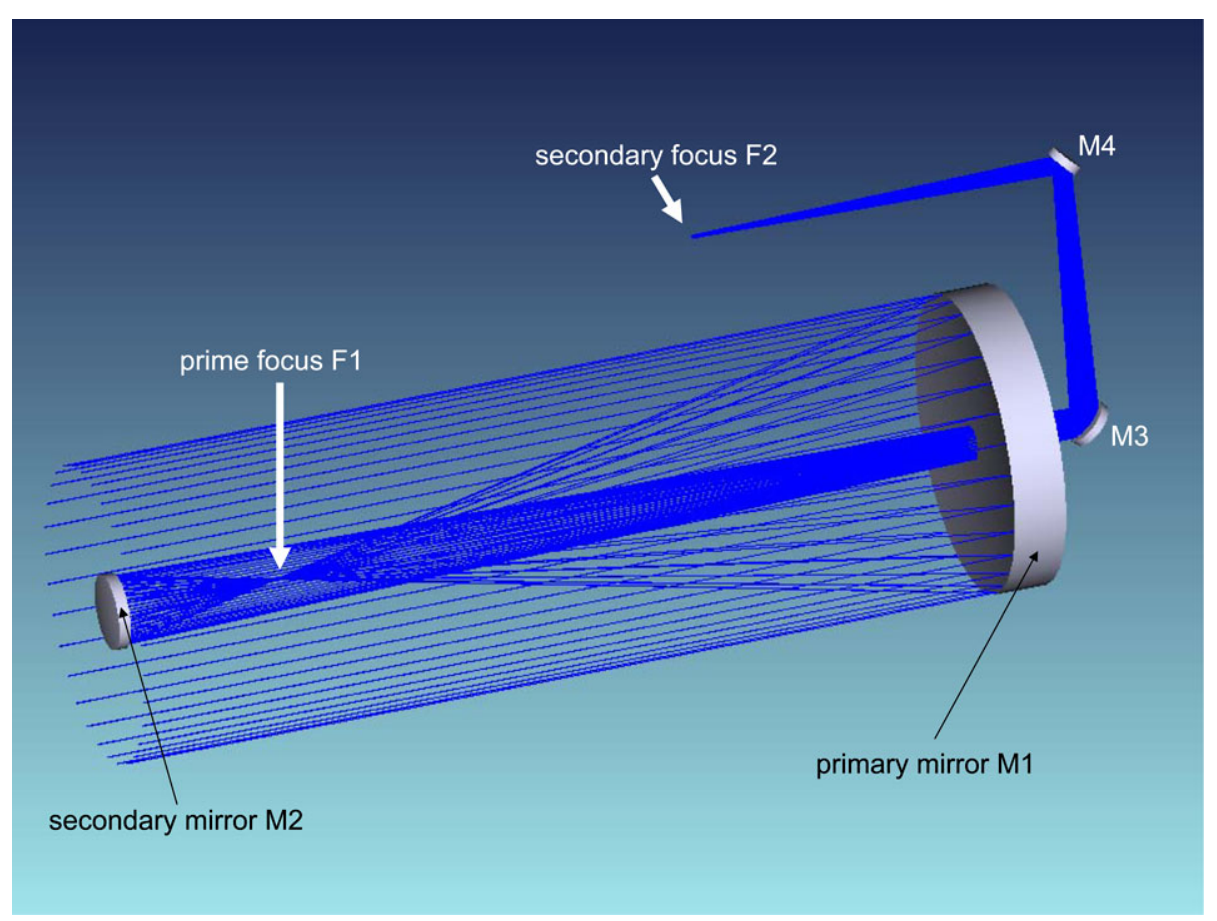

Figure 2 Optical configuration of the 1-m Gregory telescope of Sunrise.

\subsection{Telescope}

The Sunrise telescope is a light-weight Gregory-type reflector with nearly $25-\mathrm{m}$ effective focal length. The optical path is depicted in Figure 2. A parabolic primary mirror with 1010-mm outer diameter and 1000-mm clear aperture creates a real image of the sun in the first focus F1 of the telescope, $2422.9 \mathrm{~mm}$ in front of the M1 vertex. A field stop in F1 limits the telescope field-of-view (FoV) so that only a small fraction reaches the elliptical secondary mirror M2. M2 has a diameter of $245 \mathrm{~mm}$ and a focal length of $505 \mathrm{~mm}$. M2 magnifies the image in F1 by a factor of ten and refocuses the radiation to a secondary telescope focus, located in the postfocus instrumentation package on top of the central telescope frame. Two plane fold mirrors M3 and M4 redirect the light from the telescope to this upper level. A further physical stop in the secondary focus limits the telescope FoV to 180 arcsec.

The Serrurier structure (Figure 3) connects the steel central frame, the front ring with the secondary mirror assembly and heat rejection wedge, and the rear ring with the primary and tertiary mirrors. The central frame serves as the main structural component. It is a riveted sheet metal construction with metallic inserts at locations with high mechanical load. The frame carries the postfocus instrumentation (PFI) piggy-back on top. Axles mounted left and right to interface plates at the height of the combined telescope and PFI center-of-gravity form the elevation axis that connects to the gondola structure. The front ring carries the housing of the secondary mirror assembly and the heat rejection wedge including radiators. On its front side facing towards the Sun, two Sun sensors are mounted. The LISS (Lockheed Intermediate Sun Sensor) and Full Range Elevation Device (FRED) are part of the gondola pointing and tracking control system (Section 2.3.3), with the LISS serving as pointing 


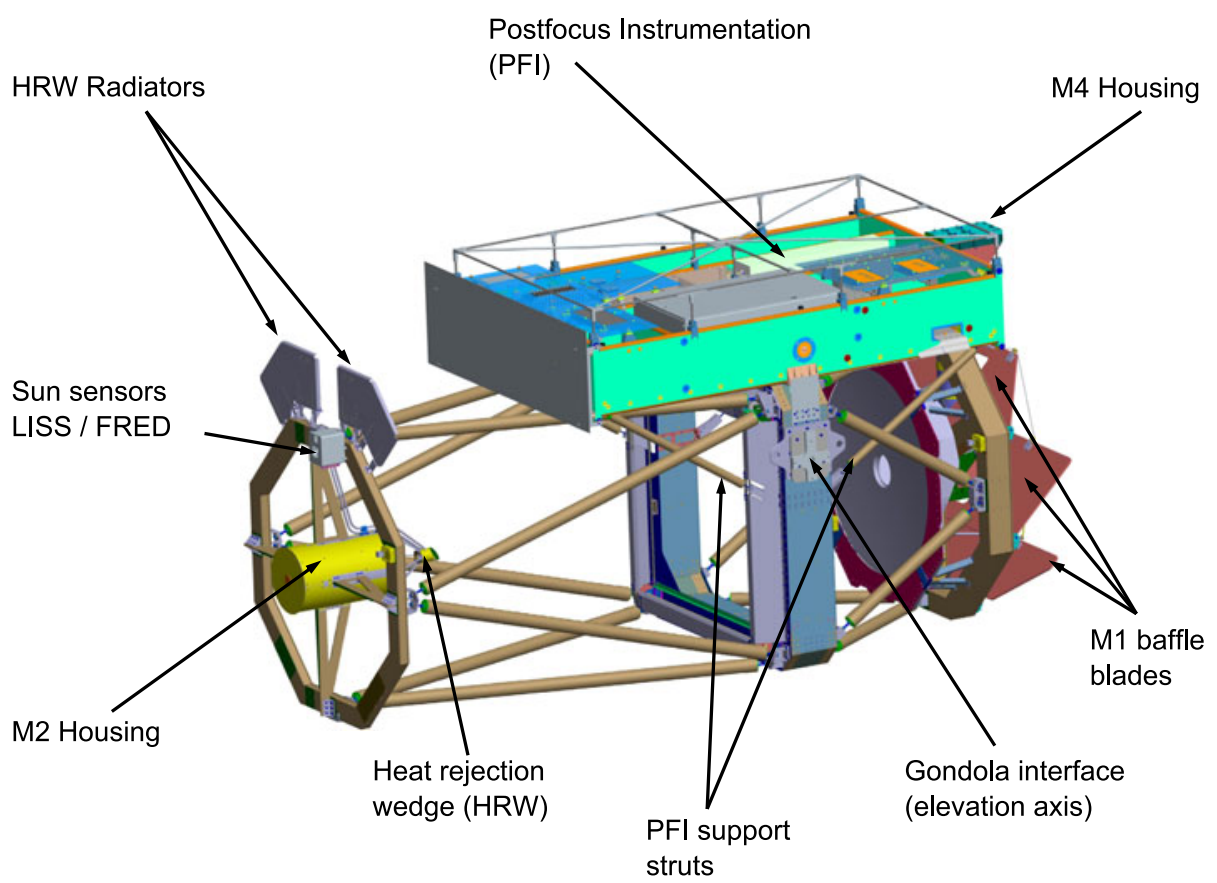

Figure 3 Overall sketch of the telescope Serrurier structure and subassemblies.

reference in flight. The back ring (see also Figure 4a) provides a stiff platform for the primary mirror and aperture stop. On its rear side, the tertiary plane fold mirror is mounted, as well as three blades providing thermal control of the primary mirror. Front and rear ring are connected to the central frame with eight struts each, forming the characteristic Serrurier mounting. This mounting maintains parallelism of the main elements even in case of relative lateral displacements due to changing gravity loads. The rings as well as the connecting struts are made of carbon fiber reinforced plastic composite materials for high stiffness, low weight and low thermal expansion. The stiffness of the struts is designed such that front and rear ring show approximately the same lateral displacement in the presence of gravitational loads, keeping the relative positioning of M1 and M2 within fractions of a millimeter. The connection of the struts to the central frame was made by steel shafts, which allowed easy and quick dismantling of the main telescope parts in the field after recovery.

The main mirror has been manufactured by SAGEM in France. Schott grade 0 Zerodur is used as substrate material. The rear side of the mirror has a three-fold symmetry with triangular honeycomb structuring, optimizing stiffness under varying gravitational loads (Figure $4 \mathrm{~b}$ ). Its thickness is $178 \mathrm{~mm}$ with a weight of less than $47 \mathrm{~kg}$. This extreme lightweighting is achieved by mechanical milling and acid etching. Three Invar mounts connect the mirror with the rear ring of the telescope structure (Figure 4a). On each mount, the glassmetal junction is formed by three Invar pads of $25-\mathrm{mm}$ diameter, which are glued to the structure behind the face sheet (Figure $4 \mathrm{c}$ ). The facesheet of the mirror was ground and polished in a conventional way. As final surface treatment step, ion-beam figuring was applied. This technique removes residual quilting, which is unavoidable when manufacturing lightweighted mirrors with facesheet thicknesses in the order of $7 \mathrm{~mm}$. To further improve the overall wavefront quality, a dedicated map-imprint was applied during the ion-beam 


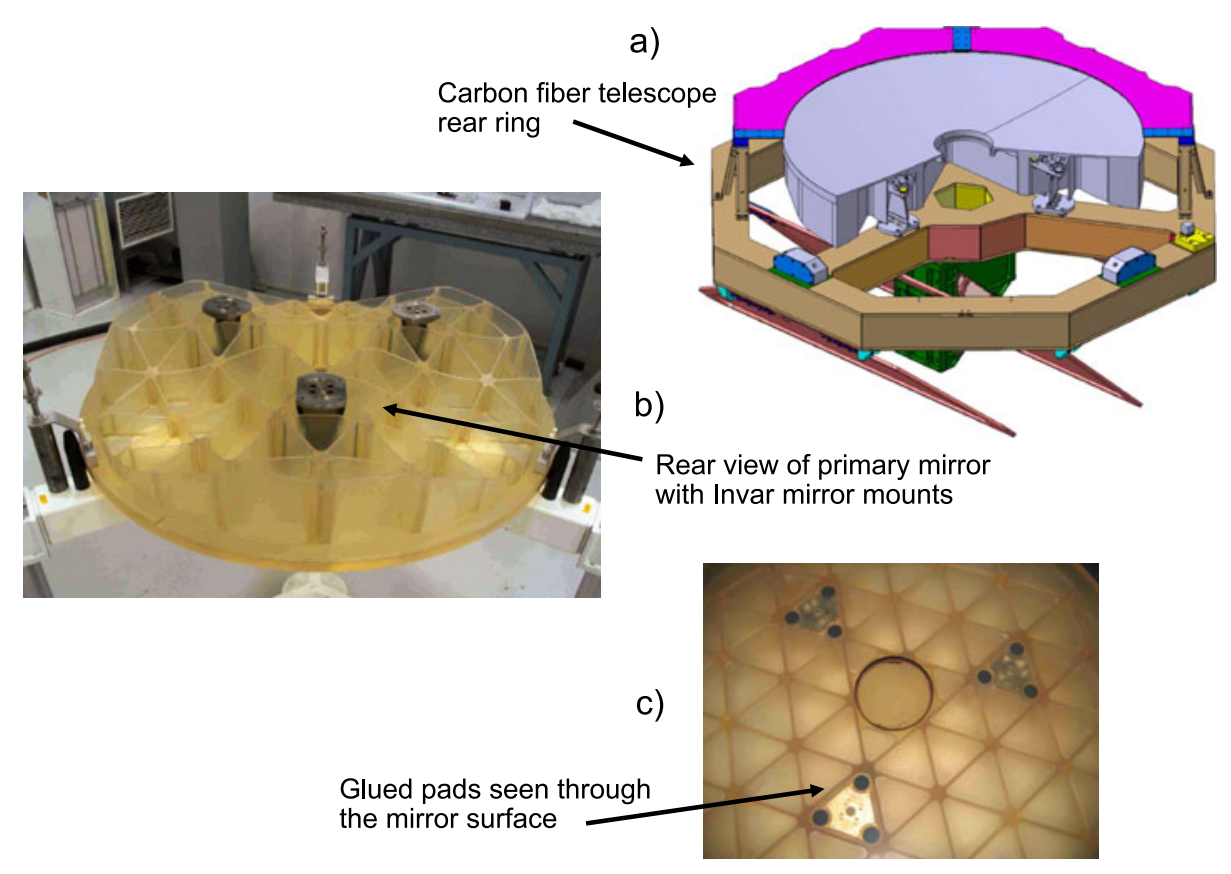

Figure 4 (a) CAD model of the main mirror cell with the lightweighted ZERODUR mirror. The mirror is mounted with three flex blade fixation points to the carbon fiber structure, which forms the rear ring of the telescope. (b) Backside view of main mirror after integration of the three flex blade fixation points. (c) Glass/metal junction formed by glued Invar pads.

figuring. Based on finite-element mechanical deformation calculations and analysis of the various thermal load cases, a compromise was calculated providing best wavefront error (WFE) performance at $22.5^{\circ}$ elevation, with only minimal degradation in the range from $0^{\circ}$ to $45^{\circ}$ elevation angle, which is the nominal elevation range of Sunrise. The measured WFE (@633 nm) at $0^{\circ}$ elevation is only $30.2 \mathrm{~nm} \mathrm{rms}$, reducing to $19.2 \mathrm{~nm} \mathrm{rms}$ at $22.5^{\circ}$ and $24.1 \mathrm{~nm} \mathrm{rms}$ at $45^{\circ}$ elevation. The reflective coating on the front face is bare aluminium with a thickness of $100 \mathrm{~nm}$. An aperture stop with 1000-mm inner diameter directly in front of M1 defines the entrance pupil of the system.

The primary mirror creates a real image of the sun in the primary focus F1 of the telescope. Nearly $1 \mathrm{~kW}$ solar radiation is concentrated on a disk of about $22-\mathrm{mm}$ diameter. At this position a heat rejection wedge (HRW) with a central hole acts as field stop (Figure 5).

The 2.8-mm hole - and a slightly undersized stop in the conjugate image plane in F2 - define the useable telescope field-of-view (FoV), corresponding to $130 \mathrm{Mm}$ on the solar surface. The HRW is a highly conductive cylindric aluminium block with a wedged front face reflecting $99 \%$ of the incoming light out of the telescope. The small fraction of solar radiation passing through the field stop reduces the heat load on the science instrumentation to about $10 \mathrm{~W}$. Absorption of the focused energy at the HRW is minimized by a second surface mirror, glued to the aluminium body. A thin glass plate with vapor deposited aluminium on the rear side acts as optical solar reflector; UV reflectivity is enhanced by a dedicated coating on its front surface. The size of the HRW is about twice the diameter of the solar disk image, thus allowing solar limb observations, while keeping the solar image on the HRW. Two ammonia heatpipes connect the HRW to dedicated radiators. Although directly oriented 

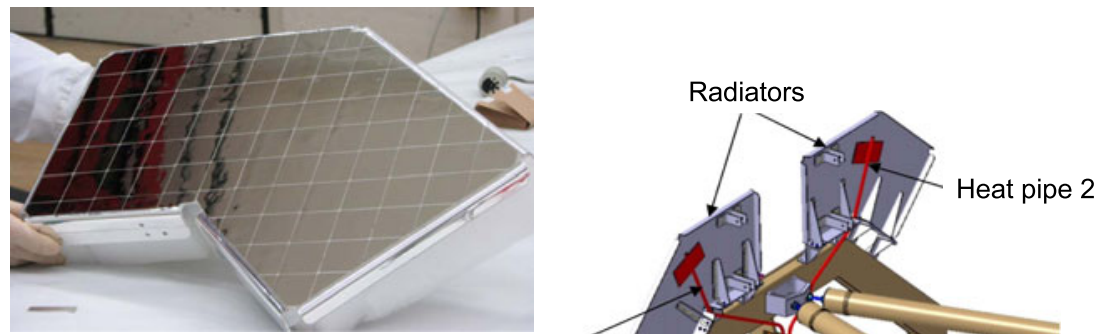

Heat pipe 1
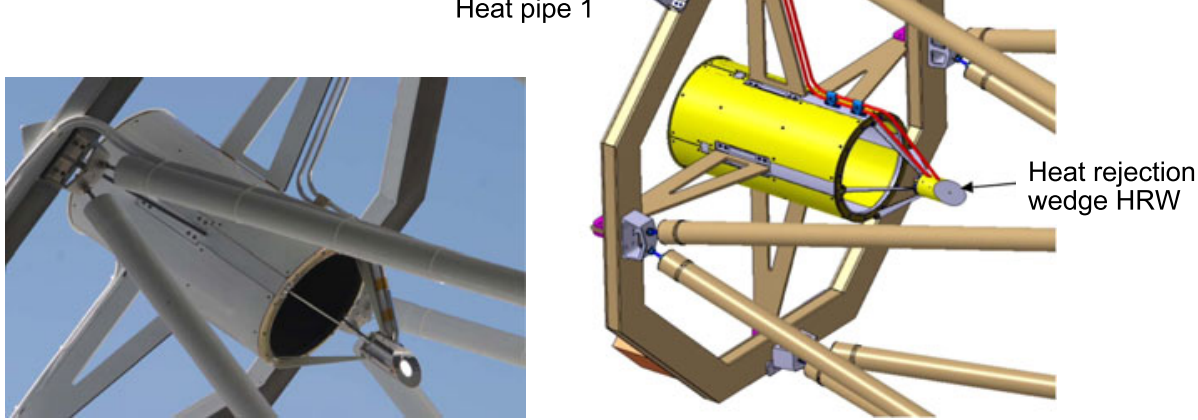

Figure 5 CAD model of the primary field stop (heat rejection wedge, HRW) with the two heat pipes, which drain the absorbed energy to the two radiators on top of the front ring. The radiators are facing direct sunlight and are therefore covered with optical solar reflectors (OSRs), thin second surface mirrors with high thermal emissivity, as shown in the insert on the top left. The bottom left picture shows the image of the solar disk on the HRW during ground testing.

towards the Sun, these radiators efficiently cool the HRW to temperatures less than $25^{\circ} \mathrm{C}$, thus avoiding Schlieren build-up, which could cause wavefront deformations. The radiators are equipped with optical solar reflectors - same second surface mirrors, but without UV coating -, providing very low solar absorptance and high emissivity in the infrared. Their orientation maintains a very stable setting with almost constant input and a large view factor to cold space, avoiding obstructions by payload components or the balloon. Temperature variations due to the changing environment and telescope elevation angles are minimized.

The optical system of the Sunrise telescope is semi-active in order to maintain the highest performance throughout the flight. The secondary mirror M2, polished and ion-beam figured to a residual WFE below $6 \mathrm{~nm} \mathrm{rms}$, is isostatically mounted on bipods and connected to a three-axis translation stage. The M2 position is fine adjustable in a range of $1.2 \mathrm{~mm}$ to an accuracy of $5 \mu \mathrm{m}$ laterally and $1 \mu \mathrm{m}$ axially, so that the relative M1/M2 alignment can be kept constant even under varying telescope elevation and thermal loads. A wavefront sensor located in the postfocus instrumentation (see below) monitors the alignment status and generates control signals for M2 mirror repositioning. The secondary mirror assembly with its housing creates a central obscuration of $324 \mathrm{~mm}$ diameter for the telescope.

The folding mirrors M3 and M4 are equipped with translation stages. It was originally foreseen to use them for fine focussing. However, the adjustment accuracy of the M2 axial translation stage proved to be sufficiently high, so that focussing could be performed with M2. The WFE increase due to spherical aberration when axially moving M2 is negligible. M3 and M4 are only adjusted during static alignment on ground, determining beam height and secondary focus position within the PFI as well as the lateral position of the pupil image at the image stabilization tip/tilt mirror. 
Thermal control of the primary mirror is essential for the performance of the telescope. About $80 \mathrm{~W}$ solar radiation are absorbed in the coating. Dedicated baffle blades behind the mirror with reflective front sides increase the view factor to the cold sky and shade the mirror against Earths IR radiation and reflected sunlight from the ground (ice) or sea below the balloon.

The energy density in the primary focus is high enough to damage structural parts in case of uncontrolled beam wandering. A retractable curtain in the plane of the central frame can close the rear compartment of the telescope in case of pointing loss, acting as an aperture door. The curtain needs about $20 \mathrm{~s}$ to securely block the telescope aperture. This is well below critical exposure times of parts in the vicinity of the first field stop. In-flight operation of the curtain is controlled by two software flags set by the pointing system computer. Automatic closure is initiated when the pointing error exceeds \pm 15 arcmin (coarse pointing flag 'off', solar disk image could leave heat rejection wedge). Re-opening is initiated if the pointing remains stable within a cone of 20 arcsec (fine pointing flag 'on', pointing is within range of image stabilization).

The telescope has been built by Kayser-Threde, Munich, under contract of MPS.

\subsection{Postfocus Instrumentation Platform}

The Sunrise Postfocus Instrumentation (PFI) rests piggy-back on top of the telescope. The compact package consists of a rigid support structure and four instrument modules with their supporting proximity electronics (Figure 6). Two of the four instrument modules are 'service' units: the Image Stabilization and Light Distribution (ISLiD) and Correlation tracking and Wavefront Sensing (CWS) units. The science instrumentation consists of the Sunrise

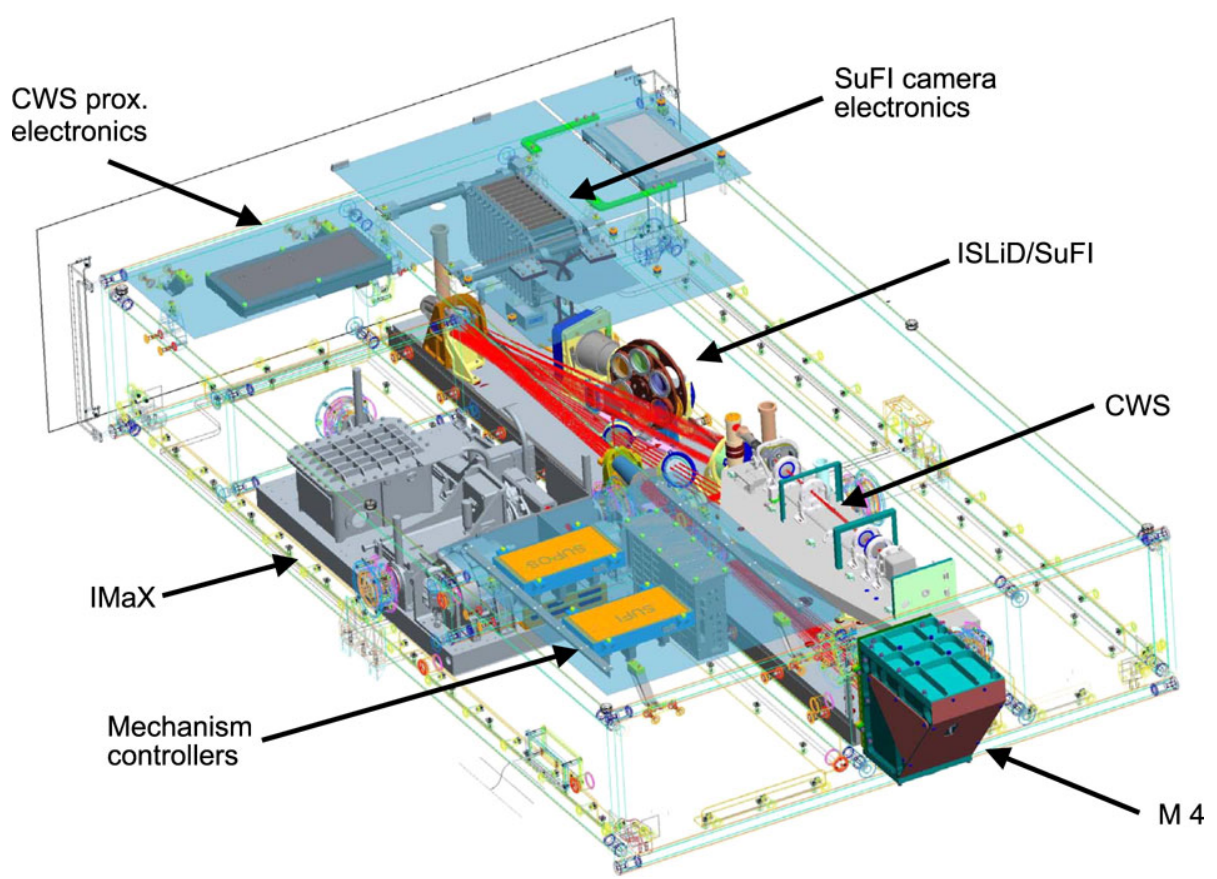

Figure 6 Semi-transparent view of the Sunrise postfocus instrumentation. Instrument covers are removed. 
Filter Imager (SuFI), and the Imaging Magnetograph Experiment (IMaX). Mechanism controllers for ISLiD and SuFI as well as proximity electronics and power supplies for CWS and SuFI are located inside some of the PFI compartments. The PFI structure provides room for an additional third science instrument for future flights.

\subsubsection{Image Stabilization and Light Distribution System: ISLiD}

The Sunrise science requirements demand precision fine pointing and simultaneous observations by all science instruments. This is ensured by ISLiD, a panchromatic reimager based on dichroic beamsplitters, which provide the different wavelength bands to the individual science branches with maximum photon flux, while preserving the polarization of the incoming light. ISLiD is located in the center of the PFI structure and takes up its full length (Figure 7). Reimaging of the secondary telescope focus onto the instrument detectors is achieved with a two mirror Schwarzschild arrangement, before separating the ultraviolet below $400 \mathrm{~nm}$ towards SuFI. Additional refractive optics provide image scaling and telecentricity for IMaX and CWS, which is fed by light outside the spectral bands used by the science instruments. ISLiD contains a fast piezo-driven tip/tilt mirror at a pupil plane of the optical system. A field lens in the telescope secondary focus projects the aperture stop of the primary mirror onto the tip/tilt mirror. In order to allow for the UV part of the solar spectrum to be transmitted, the field lens is made from fused silica and uncoated. The tip/tilt mirror is part of the CWS system (see below) and used to compensate for residual image motion due to solar rotation or gondola shake and vibrations within the instrument. The sizing of telescope and instrument FoV's allows a capture range for the image stabilization system of \pm 46 arcsec (Figure 8), still avoiding vignetting instrument FoV's by the secondary field stop in F2. Details of ISLiD (developed by MPS) are given by Gandorfer et al. (2010). The image stabilization system and its damping characteristics are described in Berkefeld et al. (2010) and in the following section.

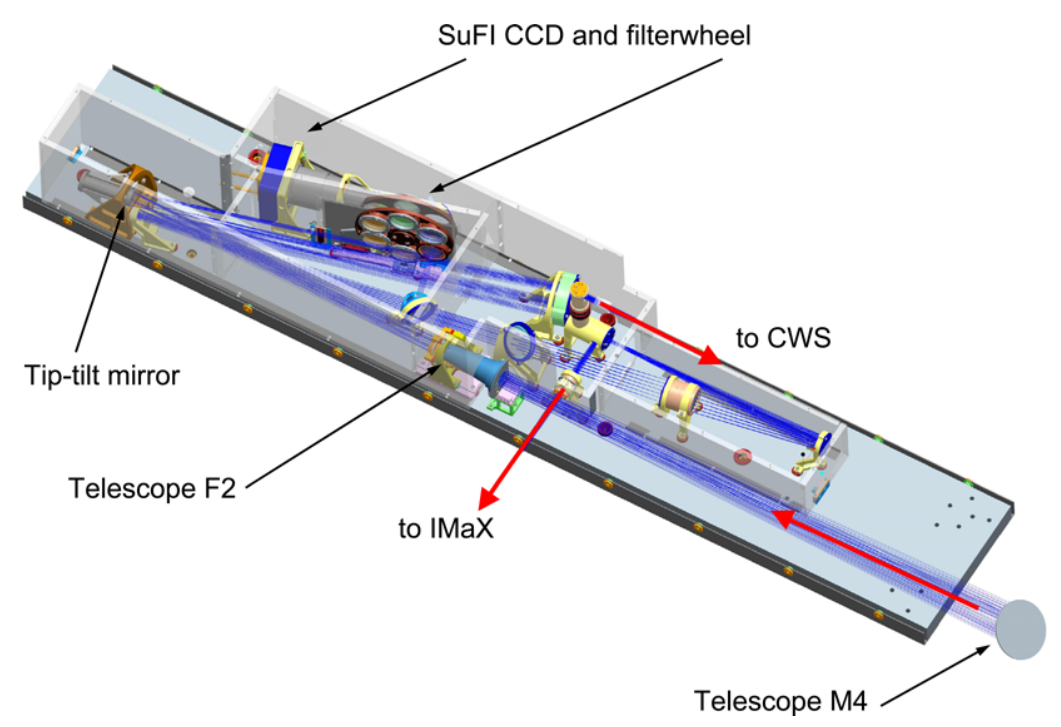

Figure 7 The Image Stabilization and Light Distribution unit (ISLiD) is mounted into the central compartment of the PFI structure. It distributes the light coming from the telescope (directions shown in red) onto the science instruments and the CWS. 

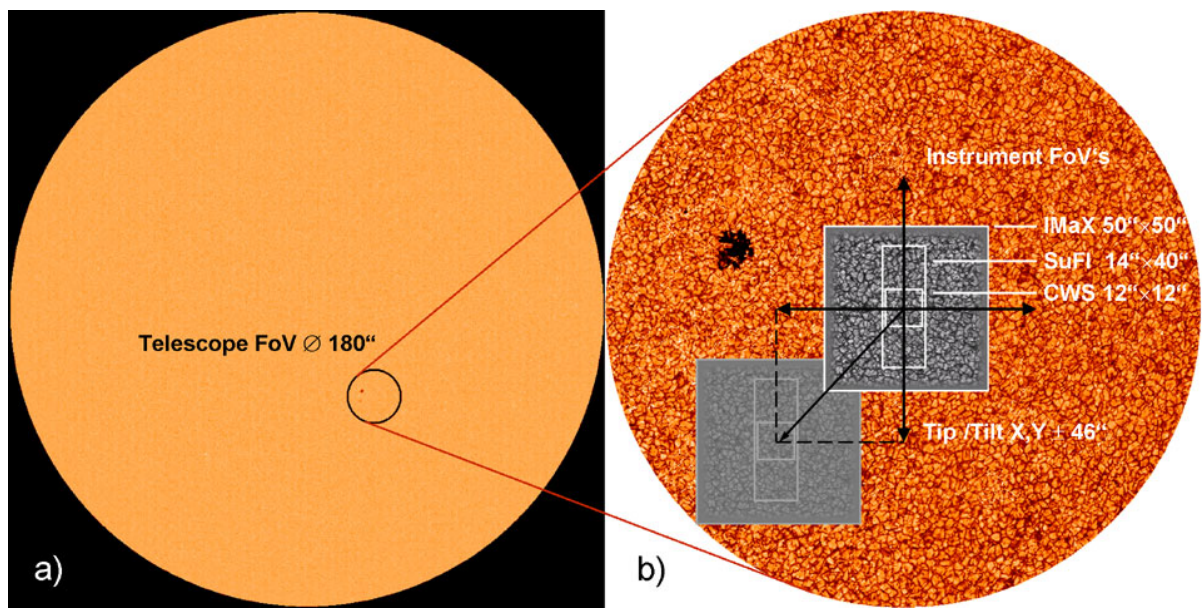

Figure 8 Telescope and instrument fields-of-view (FoV). (a) Continuum image of the solar disk. A circle indicates the Sunrise telescope FoV of 180 arcsec diameter, corresponding to roughly $10 \%$ of the solar disk diameter. (b) Co-aligned instrument FoV's of IMaX, SuFI and CWS within the telescope FoV. The free range of the image stabilization system $( \pm 46 \mathrm{arcsec})$ is indicated.

\subsubsection{Correlating Wavefront Sensor: CWS}

The CWS, a Shack-Hartmann type wave front sensor, is located close to ISLiD in the central compartment of the PFI structure. The high speed camera system has a FoV of $12 \times 12 \operatorname{arcsec}^{2}$ on the sky. The CWS is used for two purposes, for (high frequency) precision image stabilization and guiding and to control the alignment of the telescope (low frequency). A lenslet array in a pupil plane has six subapertures arranged in a concentric ring, forming six independent images on the detector. The information derived from the six independently analyzed images of the same solar scene are used to measure the local wavefront tilt per subaperture, providing the coefficients of a Zernike polynomial decomposition of the wavefront error up to the third radial degree. The coefficients for tip and tilt, defocus, and Seidel coma are used as error signals. A control loop time-integrates these error signals and converts them into actuation signals to drive the telescope secondary mirror M2. The fast read-out of the CWS camera ( $>1 \mathrm{kHz}$ ) allows detecting correlated image motion of the six separately generated images on the detector caused by residual uncompensated gondola movements and vibration as well as by the slow drift of solar features due to solar rotation. Fast software routines convert the correlation signals to actuator signals for the tip/tilt mirror, performing the pointing correction, image stabilization and guiding. The closed-loop control of the image stabilization system has a bandwidth of about $60 \mathrm{~Hz}$ (at the $6 \mathrm{~dB}$ level) with a sensitivity of better than 0.003 arcsec. It provides very efficient damping of low frequencies $(\approx 600$ at $2 \mathrm{~Hz}, \approx 70$ at $10 \mathrm{~Hz}$ ), where the pointing control cannot be provided solely by the gondola. The CWS was activated whenever the gondola pointing was within its nominal range and the pointing system software flag 'PS-lock' was set (see Section 2.3.3). The CWS unit including the tip/tilt mirror, electronics and the control software were developed by the Kiepenheuer-Institut für Sonnenphysik, Freiburg, Germany. Details can be found in Berkefeld et al. (2010). 


\subsubsection{Sunrise Filter Imager: $\mathrm{SuFI}$}

SuFI provides high-resolution images of photospheric and chromospheric features at five wavelength bands in the visible and near ultraviolet at high cadence. The optical arrangement is a Schwarzschild two-mirror system (part of ISLiD), magnifying the telescope secondary focus by a factor of five onto the detector, thus resulting in an effective focal length of $121 \mathrm{~m}$. The sensor is a $2 \mathrm{k} \times 2 \mathrm{k} \mathrm{UV}$ enhanced fast read-out CCD. The field-of-view of the instrument is $15 \times 40 \operatorname{arcsec}^{2}$. Interference filters are employed to isolate the observed wavelength band. They have peak transmissions at wavelengths of 214 (10) nm and 300 (5) nm together with $\mathrm{OH}$ and $\mathrm{CN}$ molecular absorption bands at $312(1) \mathrm{nm}$ and $388(0.8) \mathrm{nm}$ and the Ca II H line (singly ionized calcium) at $397.6(0.18) \mathrm{nm}$. The numbers in brackets are the filter FWHM. In order to achieve near diffraction-limited imaging, a phase-diversity imaging technique is used by splitting the image in front of the CCD detector: half of the CCD area collects the focused image, while an optical delay line forms a second image with a defocus of approximately one wave on the other half of the CCD. Post-flight restoration of the images free from static aberrations of the optical path can thus be achieved. The SuFI optical elements, mechanisms and CCD are mounted side-by-side with ISLiD components on one combined optical bench in the central compartment of the PFI structure. Details of the SuFI instrument, which has been developed by MPS, are given by Gandorfer et al. (2010).

\subsubsection{Imaging Magnetograph eXperiment: IMaX}

IMaX is an imaging vector magnetograph for observations of Doppler shifts and polarization in the Zeeman-sensitive photospheric spectral line of neutral iron at $525.02 \mathrm{~nm}$. Images are taken in up to 12 wavelength bands. The instrument provides fast-cadence, high-spatial resolution two-dimensional maps of the magnetic vector, the line-of-sight velocity, and continuum intensity. It has a field-of-view of $50 \times 50 \mathrm{arcsec}^{2}$, which is the largest of all instruments on Sunrise. A tunable $\mathrm{LiNbO}_{3}$ solid state Fabry - Pérot etalon is used in double pass, thus minimizing mass and power consumption and relaxing the requirements on passband stability. Since the free spectral range of such a system is quite small, a narrowband interference filter (FWHM $0.1 \mathrm{~nm}$ ) is used in addition. Both, prefilter and etalon, are thermally stabilized. Imaging is done with two synchronized $1 \mathrm{k} \times 1 \mathrm{k}$ CCD cameras in orthogonal polarization states. Two nematic liquid crystal retarders modulate the incoming polarization. Four switching states are needed for full Stokes vector polarimetry, while two-states are sufficient for longitudinal magnetometry (circular polarization). IMaX was developed by a Spanish consortium led by the Instituto de Astrofísica de Canarias, La Laguna (Tenerife), in cooperation with the Instituto de Astrofísica de Andalucía, Granada, the Instituto Nacional de Tecnicas Aeroespaciales, Madrid, and the Grupo de Astronomia y Ciencias del Espacio, Valencia. The instrument is described by Martínez Pillet et al. (2010).

\subsubsection{PFI Support Structure}

The PFI structure shown in Figure 9 provides a stable platform for the science instrumentation. The individual instruments must be kept co-aligned to better than $\pm 0.1 \mathrm{~mm}$. This is a demanding requirement considering instrument masses close to $50 \mathrm{~kg}$ (in case of IMaX) and in view of the varying gravity and thermal loads during the mission. In addition, the combined PFI package has to maintain the alignment with respect to the telescope exit beam entering the PFI via M4. Similar to the telescope, carbon fiber based composites and honeycomb structures are used for the mechanical frame. This choice provides high stiffness 

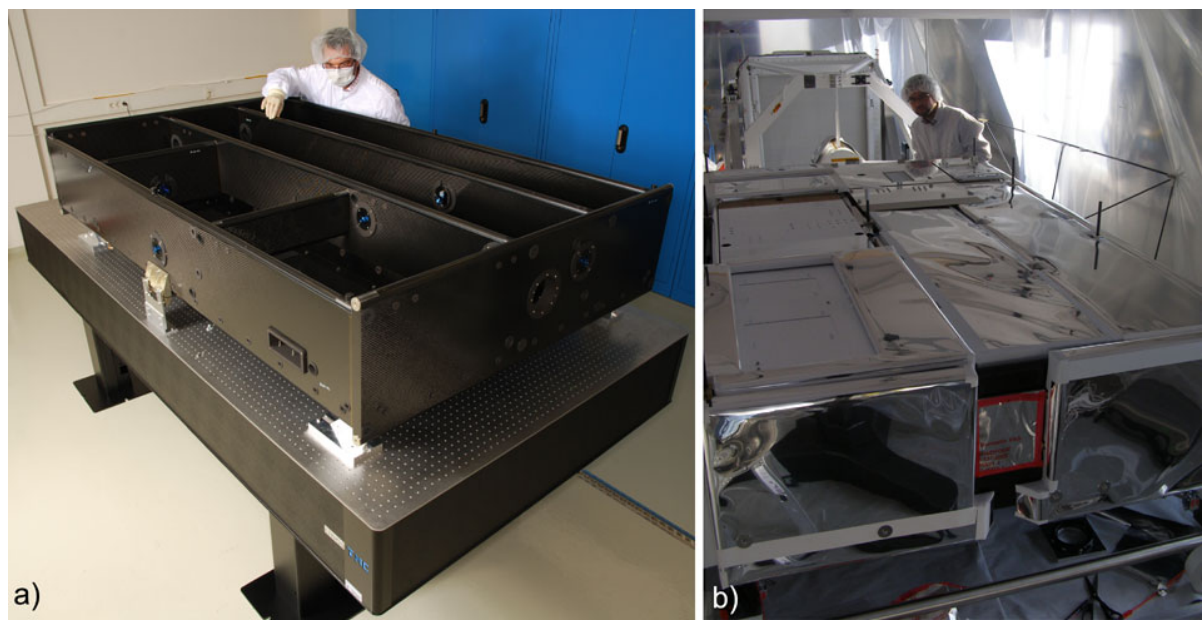

Figure 9 (a) Integrated PFI Support Structure without instrumentation mounted on optical bench, attached at all seven interface points. (b) Fully assembled PFI in thermal insulation ready to be integrated onto telescope.

and low thermal expansion, together with low weight. The PFI structure has a width of approximately $1.4 \mathrm{~m}$ (compatible with the width of the telescope) and a length of about $2 \mathrm{~m}$, suitable to accommodate the instruments. The height of $350 \mathrm{~mm}$ resulted from a trade-off between keeping the PFI center-of-gravity as low as possible and taking advantage of the increasing bending and torsional stiffness with height.

Four outer panels form a box-like structure. Two inner panels spanning the full length of the platform provide additional support for the inner instruments and for the plane folding mirror M4, which is attached to the rear PFI panel. Each panel is a combination of a $30 \mathrm{~mm}$ thick aluminium honeycomb core with carbon-fiber-reinforced plastic face sheets of $1.25 \mathrm{~mm}$ thickness. Dimensional stability of the panels is provided by a $\left( \pm 45^{\circ}, 0^{\circ} / 90^{\circ}\right.$, $\pm 45^{\circ}$ ) orientation of the individual Torayca M40J fiber-layers of each facesheet. The coefficient for thermal expansion (CTE) of the material is on the order of $-0.3 \times 10^{-6} \mathrm{~K}^{-1}$. To increase bending stiffness each panel is reinforced at the two long sides by $30 \mathrm{~mm}$ diameter, $1.5 \mathrm{~mm}$ thick tubes with unidirectional fiber orientation. Tenax UMS2526 fibers with an exceptionally high tensile strength of $190 \mathrm{GPa}$ are used.

Additional cross panels stiffen out the compartment for the heavy IMaX instrument. Shear stability of the box is provided by two panels of $10 \mathrm{~mm}$ thickness, closing the bottom end towards the telescope. A central carbon fiber based stiffener forms the central mounting point to the telescope central frame. All connections are realized by steel screws and twoparts aluminium inserts, which are inserted into the sandwich panels from both sides and secured by glue. The total weight of the PFI structure is $68 \mathrm{~kg}$ including the mounting brackets for the instrumentation.

The center-of-gravity of the PFI is located directly above the steel telescope central frame (Figure 3c). A spherical pin at the upper center of the telescope central frame is used to fix all three translational degrees of freedom (DoF). Two flexural joints at the outer uppermost ends of the telescope central frame fix the rotational DoF around the $y$-axis and $z$-axis (direction to Sun), but allowing differential thermal expansions of PFI and telescope structural elements in $x$-direction, caused by the CTE mismatch of steel and the carbon fiber matrix.

Carbon fiber based struts support all four edges of the PFI, fixing the rotational DoF around the $x$-axis (elevation axis). These struts have length compensators eliminating any 
bending forces on the PFI structure in case of temperature inhomogeneities between the telescope central frame and PFI (see Figure 3).

\subsubsection{Instrument Mounting}

All instrument units are individually assembled, aligned and functionally as well as environmentally tested as separate modules before being integrated into the PFI support structure. With the enormous effective focal length of SuFI of $121 \mathrm{~m}$ even a minimal mechanical deformation on the order of only a few micrometers already would transform into a considerable image shift on the SuFI detector. The required stability cannot be provided by the 5-cm thick optical bench alone. Increasing the overall stability of this sensitive arrangement, ISLiD/SUFI makes use of the high stiffness of the PFI support structure by being rigidly connected to the innermost side panels over its full length. L-shaped brackets connected to the lower side of the ISLiD/SuFI optical bench (Figure 7) provide a stiff mounting, but allowing for local deformations in the vicinity of the attachment points, not propagating to the surface of the optical bench or to the optical elements fixed to it. Differential thermo-mechanical expansions of the ISLiD/SuFI optical bench relative to the PFI platform are minimized by using face sheet material from the same lot for both applications.

IMaX and CWS are mounted based on an isostatic concept, allowing the individual alignment of each instrument module as such with respect to the exiting beam coming from ISLiD. This concept has the advantage that optical tolerances in focus position and exit beam orientation, as well as unavoidable manufacturing tolerances of the large PFI support structure can be easily compensated. The isostatic mounting provides a stress-free fixation of the instruments even in the case of temperature differences and materials with different CTE's. This is especially important for IMaX, which uses an aluminium optical bench.

The correct angular orientation of IMaX and CWS with respect to the exiting beams of ISLiD is achieved by the use of reflective alignment reference cubes. Dedicated observation openings in the PFI structure panels allow theodolite autocollimation measurements to arcsec precision.

A 3D coordinate measurement device (Leica Laser Tracker) led to a mounting accuracy of better than $0.1 \mathrm{~mm}$.

\subsection{Gondola}

The Sunrise gondola is responsible for the precision pointing of the telescope towards the Sun. The mechanical structure serves as stable platform and protects the scientific instrumentation during launch and landing. Photovoltaic arrays generate the required electrical power.

\subsubsection{Gondola Structure}

The core gondola structure consists of an aluminium/steel tube framework (Figure 1), making it relatively lightweight, but providing the required stiffness and a sufficiently high eigenfrequency $(>10 \mathrm{~Hz})$. The structure permits a telescope elevation range of $-5^{\circ}$ to $+50^{\circ}$, leaving some margin for pendulum motion during flight. The structure can be split in two halves at the level of the telescope elevation axis (Figure 10), resulting in two U-shaped components. This allows for a convenient integration of the science instrumentation and simplifies shipment of the bulky equipment. Each of these two units con- 


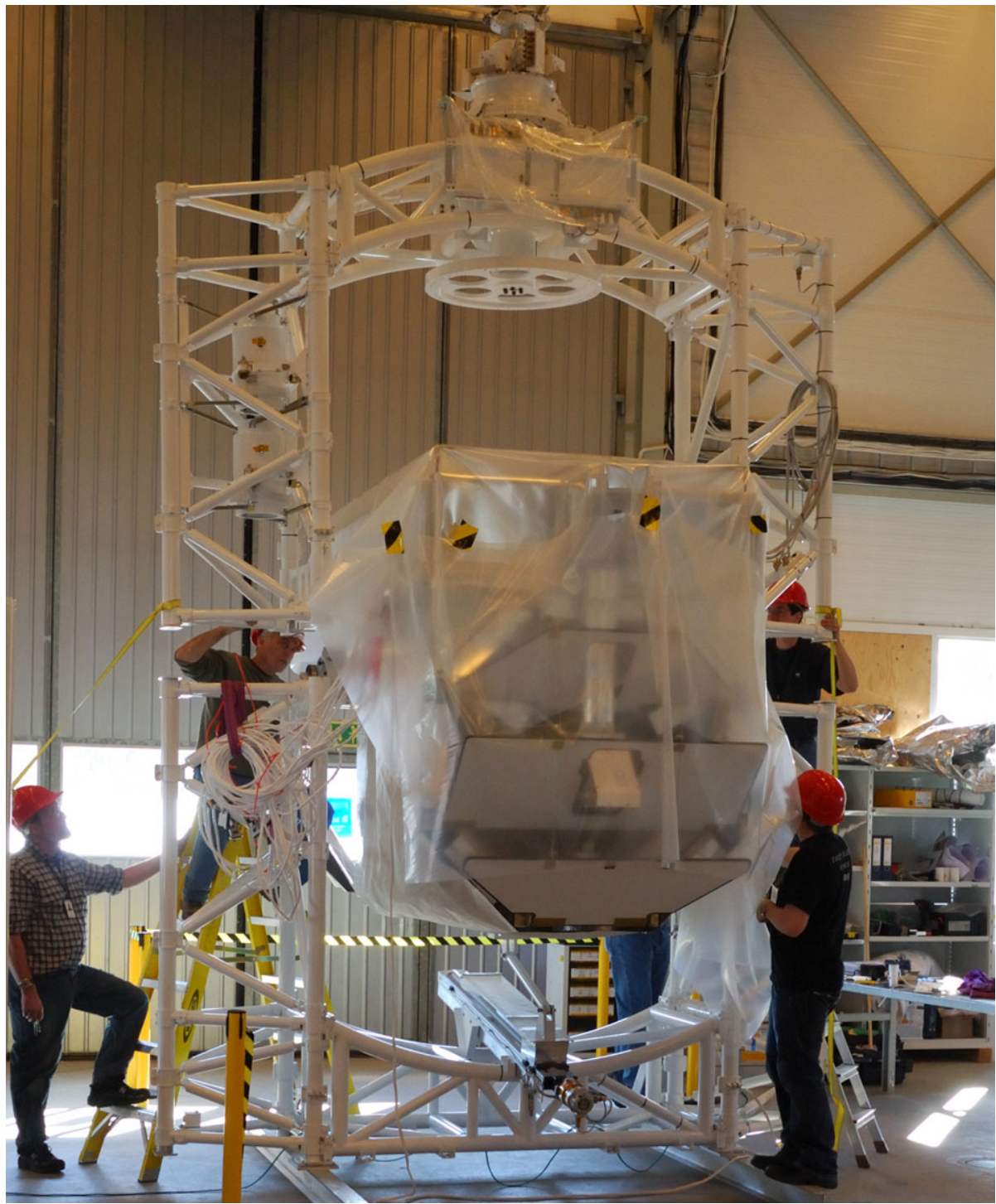

Figure 10 Sunrise gondola main structure during integration of the science payload. The two cylinders in the upper left side truss contain the science data storage systems.

sists of two aluminium side trusses with triangular cross-section; a welded steel-based bridge connects the two. Front and rear roll cages are attached to the framework structure. They protect the protruding front and rear ends of the telescope (Figure 1). Below the bottom of the framework, the crash pad assembly forms the mounting base for the cardboard landing-shock absorbers. This frame houses the CSBF provided SIP (system instrumentation package), the communication electronics needed for commanding to the instrument and balloon, as well as telemetry through TDRS and Iridium satellite links. 


\subsubsection{Power System}

Electrical power is provided by photovoltaic arrays on the front side of the gondola, sufficiently far away that the nearly $100^{\circ} \mathrm{C}$ hot panel surfaces do not generate seeing effects (Figure 1). An inclination angle of $22.5^{\circ}$ optimizes the orientation for the expected solar elevation range $\left(0^{\circ}\right.$ to $\left.45^{\circ}\right)$ during the flight. Each array consists of five subframes, each with 80 A-300 cells, produced by Sunpower Corporation. The panels, assembled by Meer Instruments, San Diego, USA, generate approximately $1.3 \mathrm{~kW}$ in total. Two lithium-ion battery packs with a capacity of 2500 Wh each act as buffers for the operation of the observatory, before the correct pointing is achieved. The battery packs are mounted into the lower bridge of the core framework.

\subsubsection{Pointing System}

The major components of the gondola pointing system are schematically displayed in Figure 11. Actuators and their encoders are shown in pale red, solar sensors in yellow.

Actuators Azimuthal control of the gondola is performed via a two-stage momentum transfer unit at the top. The coarse azimuth drive decouples the instrument from the rotating balloon, while the fine drive rotates a reaction wheel. Acceleration or deceleration of the wheel rotates the gondola towards the desired orientation. Nominal rotation rate of the reaction wheel is $10 \mathrm{rpm}$, helping to overcome friction effects in the wheel bearing. The actuation between the fine azimuth motor (the reaction wheel) and the coarse azimuth motor is divided by frequency. The coarse azimuth motor manages the frequency range below $0.1 \mathrm{~Hz}$. The reaction wheel compensates torques from $\approx 0.1$ to $\approx 1.0 \mathrm{~Hz}$. While the coarse azimuth motor has a very large compensation range (it can apply about $13.5 \mathrm{Nm}$ of torque

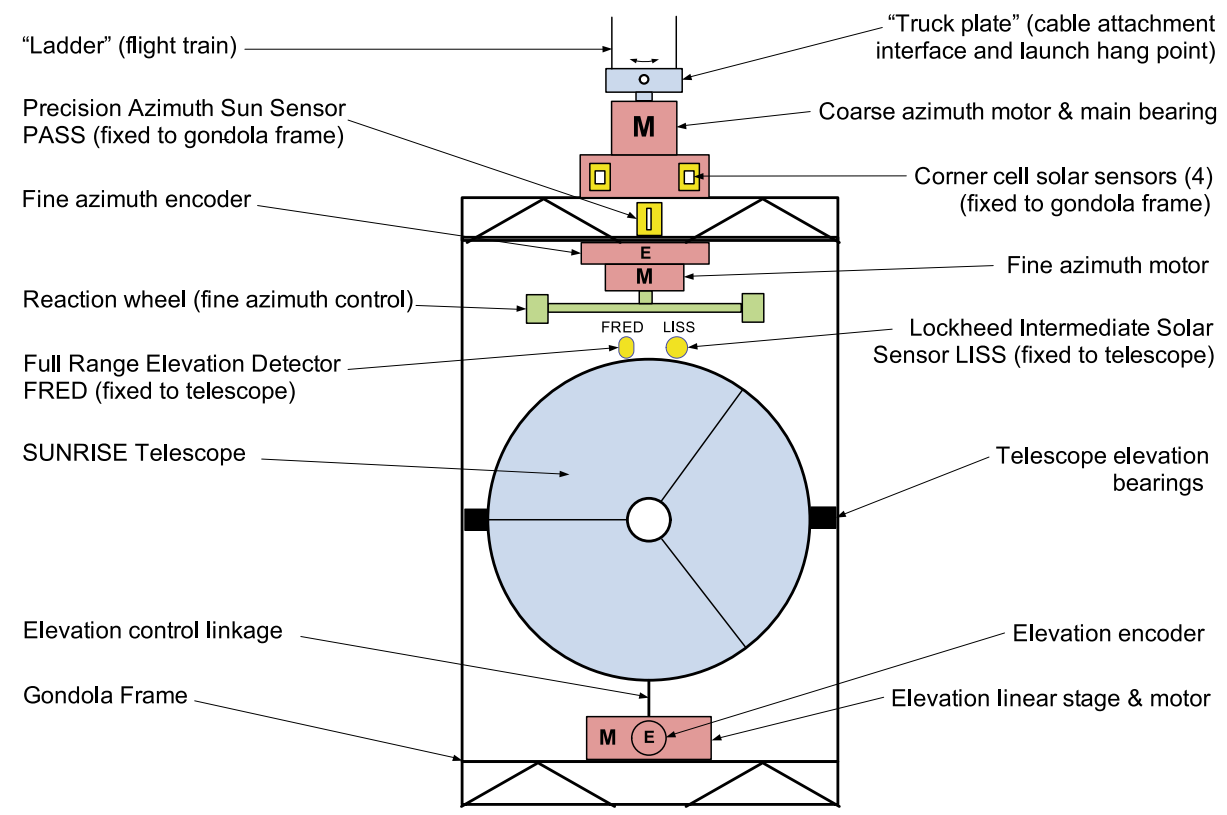

Figure 11 Basic Sunrise gondola components. 
for an indefinite period of time), the fine azimuth motor can apply an equal torque to the payload only for a limited period. Tracking is kept and the fine azimuth servo system does not saturate, as long as disturbances to the gondola - like wind gusts - induce less or equal torque for periods of less than $\approx 1 \mathrm{~s}$.

The elevation of the telescope is adjusted by a linear translation stage, rigidly coupled to the central frame of the telescope by a lever arm. Slight preloading of the lever arm minimizes hysteresis for the elevation adjustment. Several elevation drive mechanisms were considered in the early design phase, including torque motors mounted to the elevation axle. The linear stage was chosen because it provides high precision and a relatively non-compliant, symmetric loading on the central frame of the telescope. The disadvantage of this system is additional weight over a direct drive torque motor. However, the chosen solution serves as a launch and landing lock for the telescope in (horizontal) stow position, without the need of an additional mechanism.

Three types of sensors with different accuracy levels feed the various control loops used to acquire correct solar pointing.

Coarse Gondola Orientation Photovoltaic cells with wide-field, overlapping acceptance cones of $60^{\circ}$ in elevation and $105^{\circ}$ in azimuth are fixed to the gondola structure top at four corners. The right quadrant is derived from comparison of intensity levels, independent of solar elevation.

Medium Azimuth and Elevation Detection The Precision Azimuth Sun Sensor 'PASS' is also fixed to the gondola structure top. The sensor is used as medium resolution device, locating the Sun in azimuth with a linear capture range of $\pm 3^{\circ}$ for any given solar elevation. For medium resolution detection of the Sun's elevation, a similar device is used. The Full Range Elevation Detector 'FRED', mounted to the telescope front ring, can find the Sun within an azimuth range of $\pm 5^{\circ}$. This elevation sensor has a linear range of $\pm 15^{\circ}$. For angles outside this range, it gives a saturated signal indicating that the Sun is above or below the current telescope elevation. Both sensors provide accuracies of better than 10 arcsec.

High Resolution Solar Position Sensing Highest accuracy in determining the solar position is provided by the LISS (Lockheed Intermediate Sun Sensor), mounted to the Sunrise telescope front ring. The LISS is used within a $\pm 3^{\circ}$ range in azimuth and elevation, where the output signal is proportional to the off-pointing from the LISS optical axis. The high resolution linear range is only \pm 15 arcmin. Electronic noise and signal drifts with temperature are low enough, so that accuracies of 1-2 arcsec can be achieved. The LISS is mounted on a two axis motorized tip/tilt stage. Offsets in telescope pointing for solar limb observations and instrument flatfielding are generated by commanding position offsets to the LISS motors.

The medium and high resolution sensors PASS, FRED and LISS are shadow sensor type detectors. In case of the LISS, solar radiation passes a square aperture entrance window, falling on a set of five photodiodes. A central diode is used as a binary 'Sun present' indicator. The remaining four sensors are arranged in two pairs, oriented in azimuth and elevation direction. Each pair is connected to a transconductance amplifier, which amplifies the difference in photocurrent. When the sensor is tipped, the amount of light falling onto the two photocells (in a given axis) gets out of balance and produces a signal.

Acquisition of Solar Pointing The acquisition of solar pointing and tracking is completely automatic. Several software threads handle data collection, processing, actuator stimulation 
and data output generation. The 'auto-pointing thread' makes the decisions of which Sun sensors to use and what servo control loops to engage. The 'interrupt thread' runs at $150 \mathrm{~Hz}$ update rate and does all the real time pointing and data collection. It also records a boxcar array of sums and sums squared of all Sun sensor information and voltages for the elevation and coarse and fine azimuth motors. A separate thread is constantly checking thresholds to determine wether the pointing system is locked on the Sun and within the capture range of the CWS image stabilization system (see Section 2.2.2), then being indicated by the software flag 'PS-lock'.

The auto-pointing thread uses the sums and sums squared to compute running averages and standard deviations squared of these parameters. The running averages and standard deviations are computed based on the most recent 1024 values collected (1024 samples covers $6.8 \mathrm{~s}$ of data). The auto-pointing thread checks every $9 \mathrm{~s}$ all instantaneous values, running averages and running standard deviations. Then, using carefully adjusted threshold criteria, it decides which acquisition sequence needs to be activated.

The threshold criteria and activation of servos depend on which solar sensor is getting the best signal. Each pointing sequence aims to optimize the positioning for the next higher resolution sensor. The automated acquisition uses the following sequential steps with dedicated control loops:

Coarse azimuth: Coarse + fine azimuth drives to find correct azimuthal orientation based on corner cell signals, if gondola rotation rate is less than $2 \mathrm{rpm}$

Fine azimuth: Coarse and fine azimuth drives to position gondola based on PASS data

Coarse elevation: Telescope elevation being adjusted according to FRED data, until LISS sensor indicates presence of the Sun

Final tracking: Control loops closed in azimuth and elevation, based on LISS data

Final tracking is achieved by switching from 'FRED' to 'LISS' for elevation tracking and from the 'PASS' to 'LISS' for azimuth tracking. This switching in azimuth and elevation is done separately to accommodate which sensor gets to a good signal first. In azimuth the pointing system uses a 'multiple-input-multiple-output' (MIMO) approach. The final servo control loop topology is shown in Figure 12. The filters settings applied in this mode have a higher gain and are designed for a better tracking accuracy. The servo loops use a combination of integrators and phase-lead compensators with up to four filters per loop. In case of the 'AzfTrack' servo loop (see Figure 12) a combination of a second order low pass filter, then a first order lead filter and lastly a first order integrator filter is applied to the LISS azimuth output, driving the reaction wheel.

The design requirement for the tracking accuracy is \pm 7.5 arcsec rms, to safely keep the system within the capture range of the image stabilization system, provided by the tip/tilt mirror and CWS ( \pm 46 arcsec).

The automated acquisition of the Sun from a non-pointing mode to 'pointing system lock' is typically achieved within less than $10 \mathrm{~min}$.

Matlab models were used to try to predict the behaviour of the full flight train versus the suspension used during ground testing. While on ground the gondola was attached to a crane via a short rope, the flight train connecting the gondola to the balloon has a length of typically $100 \mathrm{~m}$. It consists of the parachute directly underneath the balloon and the 'ladder', a double steel cable attached to the gondola on one end and to the parachute release mechanism on the other end. The main mitigation was to not use positional feedback on the coarse azimuth drive. Such feedback gives rigid coupling to the flight train and thus the vibrational modes of the ladder would be very well coupled to the gondola. Torque feedback was used instead (simple current to the motor), assuming that the main rotator bearing 


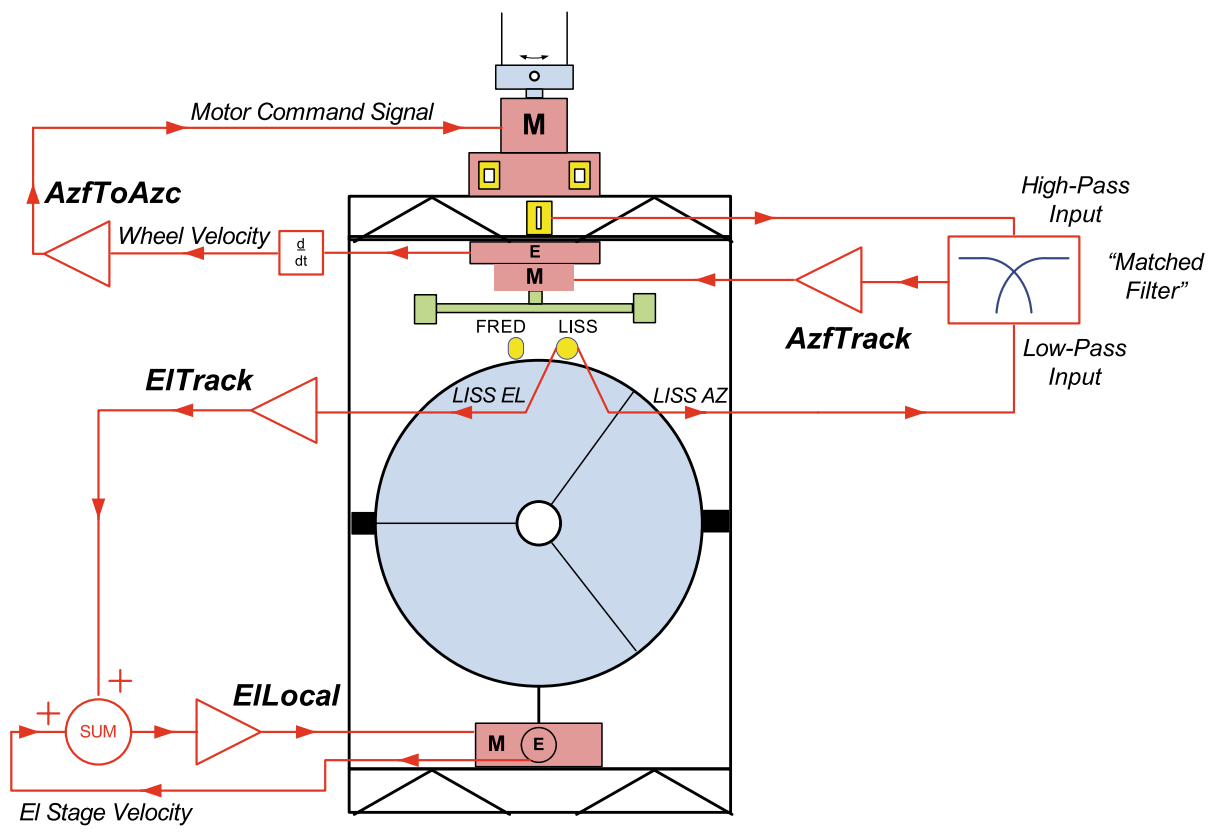

Figure 12 Sunrise final tracking servo control loop configuration. Red lines represent signal paths and triangles are signal processing (filtering). Yellow elements are solar sensors. Motors and encoders are shown in pale red. The label 'AzfToAzc' denotes the servo loop monitoring the rotational velocity of the fine azimuth drive and providing feedback to the coarse azimuth motor to eventually slow down the reaction wheel. Similarly, 'AzfTrack' and 'ElTrack' denote fine tracking servo loops. 'ElLocal' transforms input values to calibrated velocity commands for the elevation drive.

was good enough that ladder modes would be decoupled. Servo filter settings derived from the mathematical models were uploaded during the commissioning phase, being fine-tuned throughout the mission.

\subsubsection{Gondola Mounted Components/Systems}

On the rear side of the gondola, shaded by the solar panels from direct Sun illumination, the instrument control electronics are located on two racks. One rack houses the instrument control unit ICU, the payload power distribution unit and the instrument computers for CWS, IMaX and SuFI. The other rack carries the pointing system computer, the gondola power distribution unit, amplifiers for the azimuth and elevation drives, as well as the ESRANGEprovided E-Link high-speed telemetry electronics. The racks are inclined with respect to the structure in order to minimize radiative input from the Earth and the hot solar panels onto the electronics, while maximizing the radiation of heat to the cold sky above the instrument. The two data storage containers collecting data from the instruments are mounted well secured inside one of the upper side trusses of the core framework. The truss framework provides protection, but also allows easy access for recovery after landing. A spring-based shock protection system ensures the mechanical integrity of the data storage containers. To avoid oscillating masses and adverse effects on the pointing control loops, the data storage containers are rigidly clamped during the mission. A release mechanism frees the containers at mission termination. 
The commanding and communication package provided by CSBF is located underneath the gondola structure (Figure 1). It has separate solar panels at all four sides of the gondola to stay operational in case of pointing loss. The package allows commanding and housekeeping downlink via TDRS and Iridium satellites. Shock absorbing cardboard crash pads at the bottom of the gondola reduce mechanical loads during touch-down and landing. Two ballast hoppers at the center of the gondola bottom carry about $650 \mathrm{~kg}$ of fine steel grains. Part of the ballast is dropped during ascent, speeding up the balloon again after it has cooled down in the tropopause transit. The rest of the ballast is used to compensate losses in float altitude due to the day/night cycles. Two booms at the top of the gondola carry the satellite communication antennae.

The complete payload has dimensions of $5.5 \mathrm{~m}$ in width and length and is about $6.4 \mathrm{~m}$ high. The gondola structure, the power and pointing systems have been developed by the High Altitude Observatory (HAO), NCAR, Boulder, USA.

\subsection{Electronics Architecture}

The largest part of the Sunrise electronics is located on two racks mounted left and right of the gondola structure. Only proximity electronics such as mechanism controllers or the voltage supply for the piezo-driven tip/tilt mirror are located close to the optical modules inside the PFI. Commercial-off-the-shelf products are used as far as possible. These products would typically not survive the environmental conditions of a balloon flight. Critical items therefore were encapsulated in pressure vessels, modified or specifically qualified for this type of application.

Sunrise is designed for autonomous operation similar to a spacecraft. The basic architecture consists of an instrument control system communicating with dedicated subsystemor instrument-related electronics, and telemetry systems for commanding and downlink of system status information.

The instrument control system consists of the instrument control unit (ICU), two data storage subsystems, the PFI and gondola power distribution units, and the line-of-sight telemetry subsystem (E-Link).

The ICU is the central onboard computer. It supervises the various subsystem and instrument control computers:

- Pointing system computer

- Gondola power distribution unit

- CWS electronics unit

- Main telescope controller

- SuFI electronics unit

- IMaX main electronics

- Payload power distribution unit

- E-Link

- PFI mechanism controllers (also used for PFI thermal control)

The instrument control system carries out the following tasks:

- interpret and route telemetry commands to subsystem and instrument computers

- acquire housekeeping data

- store housekeeping and science image data on data storage units in RAID5 format

- pre-select and route housekeeping and science image thumbnail data to downlink telemetry subsystem 
- initialize, configure and run the Sunrise instrument automatically in standard observational mode, if no observation mode is given by telecommand

- run predefined timeline-controlled observations

- control electrical power distribution to scientific instruments and subsystems

- monitor housekeeping data and take action in case of limit violations

- provide line-of-sight telemetry

The instrument control unit (ICU) is housed in a pressurized container. It is based on a NOVA 7800 P800 Pentium III single board computer with interface cards providing ethernet and serial interfaces. A 2 GB flash disk serves as boot device. Forced convective cooling by regulated fans keeps the component temperatures within specified ranges. Onboard communication between ICU and subsystem and instrument computers is performed via a 100 base TX ethernet and serial RS232 as well as RS422 links.

The science data are stored onboard in two pressurized vessels, each containing a stack with 24 (100 GByte) harddisks. The two stacks in total provide about 3.6 Terabyte net capacity at RAID-5 functionality. This corresponds to the expected data volume acquired in a two-week mission. The harddisks are encapsuled in two pressurized vessels (see Figure 10), maintaining the required environment regarding temperature, pressure and humidity. The data from the ICU to the data storage system are transferred via IEEE1394 A (Fire Wire) links at $400 \mathrm{Mbit} \mathrm{s}^{-1}$.

The commanding and telemetry from and to the ground station is handled via the CSBFprovided system instrumentation package (SIP). The SIP provides simple pulse command channels for direct instrument control. The pulse commands are used for ICU reset control and for payload power switch off in case of emergency or to release software induced deadlocks.

\subsection{Telemetry}

Operations control for Sunrise is performed at two different locations. All balloon system relevant issues such as flight monitoring and tracking, ballast drops and mission termination are handled by the operation control center (OCC) in Palestine, Texas, USA. Sunrise science operations are directly controlled and monitored from system and instrument EGSE (electronic ground support equipment) computers at the remote operation control center (ROCC) located at ESRANGE. All command activities at ESRANGE are closely coordinated with CSBF personal present on site.

Commanding and downlink of telemetry data are provided through different communication systems depending on the mission phase.

During the first hours after launch, while the instrument still is within the line-of-sight, the E-Link high speed telemetry system is used. This communication system has been developed by ESRANGE and is available on a rental basis. It operates at frequencies around $2.4 \mathrm{GHz}$ and acts as a transparent Ethernet connection to the ICU, providing simultaneous up- and downlink rates of up to $2 \mathrm{Mbit} \mathrm{s}^{-1}$. The range is limited to approximately $350 \mathrm{~km}$. The ground station antenna tracks the instrument position autonomously using the GPS information provided onboard Sunrise. To enhance the range for line-of-sight communication, a second ground station was set up in Andenes, Norway.

'Over the horizon' communication with Sunrise is achieved by a Tracking and Data Relay Satellite System (TDRSS) communication channel. Commands generated by the Sunrise EGSE computers at ESRANGE are transferred via internet to a science relay computer at the OCC in Palestine, Texas (Figure 13). The OCC is connected with the TDRSS ground station in White Sands, New Mexico, where the Sunrise commands are uplinked to the TDRS 


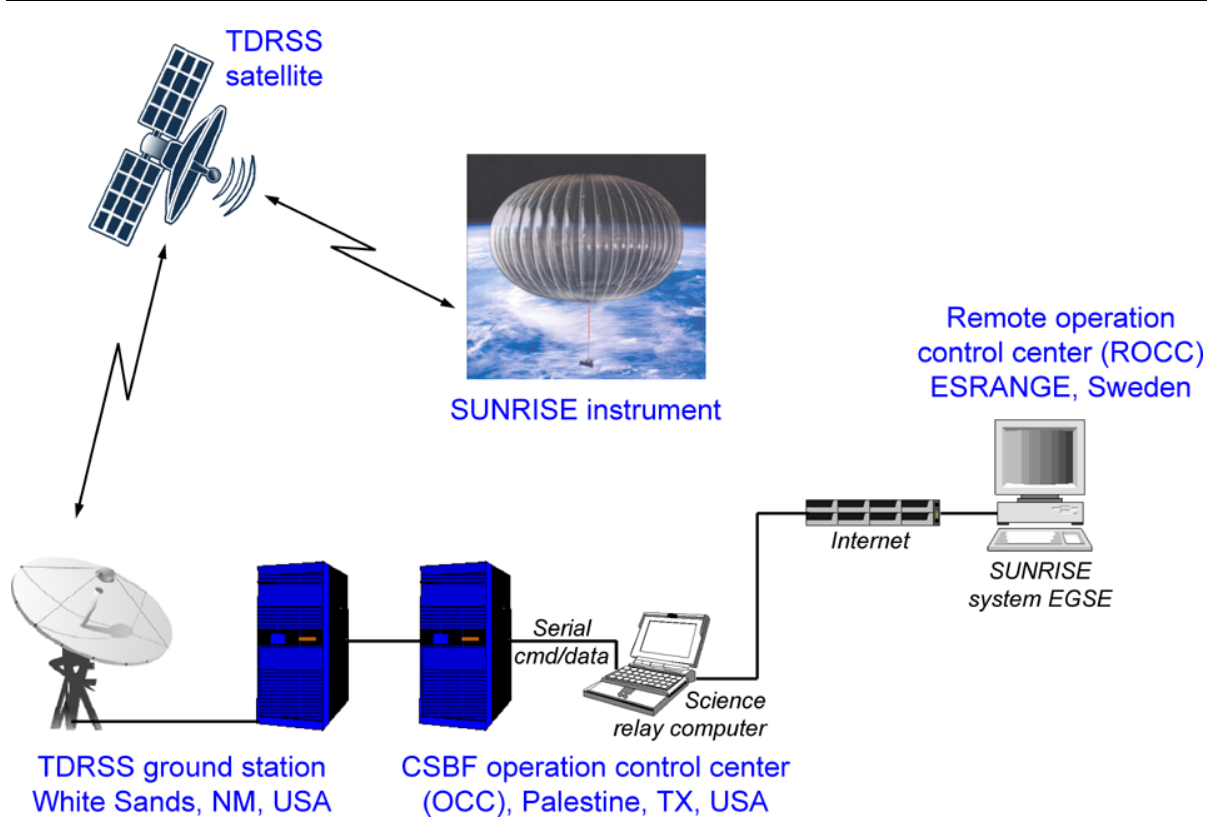

Figure 13 'Over the horizon' communication via TDRSS satellite link.

and finally relayed to the SIP antennas onboard Sunrise. The SIP transfers the commands via a serial link to the ICU. Data generated onboard, such as instrument housekeeping or thumbnail images, are relayed from the instrument back to the ROCC along the same path. A data rate of $6 \mathrm{kbit} \mathrm{s}^{-1}$ is available almost permanently for the downlink.

\subsection{Balloon System}

The science goals of Sunrise profit from as high a float altitude as possible, especially to allow observations in the UV down to $214 \mathrm{~nm}$. Therefore one of the largest balloons regularly flown by CSBF has been chosen for Sunrise, an Aerostar zero-pressure balloon with a volume of 34.43 million cubic feet $\left(975000 \mathrm{~m}^{3}\right)$ and a diameter of close to $134 \mathrm{~m}$. In case of Sunrise this balloon type lifts a total mass of about 6 tons to stratospheric altitudes of more than $37 \mathrm{~km}$. The scientific payload with a mass of $1920 \mathrm{~kg}$ contributes only one third to the total weight. The rest is given by the balloon film $(2330 \mathrm{~kg})$ with its helium filling $(500 \mathrm{~kg})$, auxiliary equipment as suspension, parachute, crush pads, ballast hoppers (Figure 14) and finally ballast $(544 \mathrm{~kg}$ ) for altitude stabilization during flight.

\subsection{System Analysis and Design Aspects}

The optical performance of Sunrise must be guaranteed under various thermal and mechanical load cases. In order to stay within the required optical tolerances, which were defined by a sensitivity and tolerance analysis on telescope and instrument level, the Sunrise design was based on a thorough analysis of the expected thermal and mechanical loadcases.

\subsubsection{Structural Design}

A structural analysis and iterative optimization of the complete instrument with all components of the gondola, telescope and PFI has been performed with the aim of maximizing 


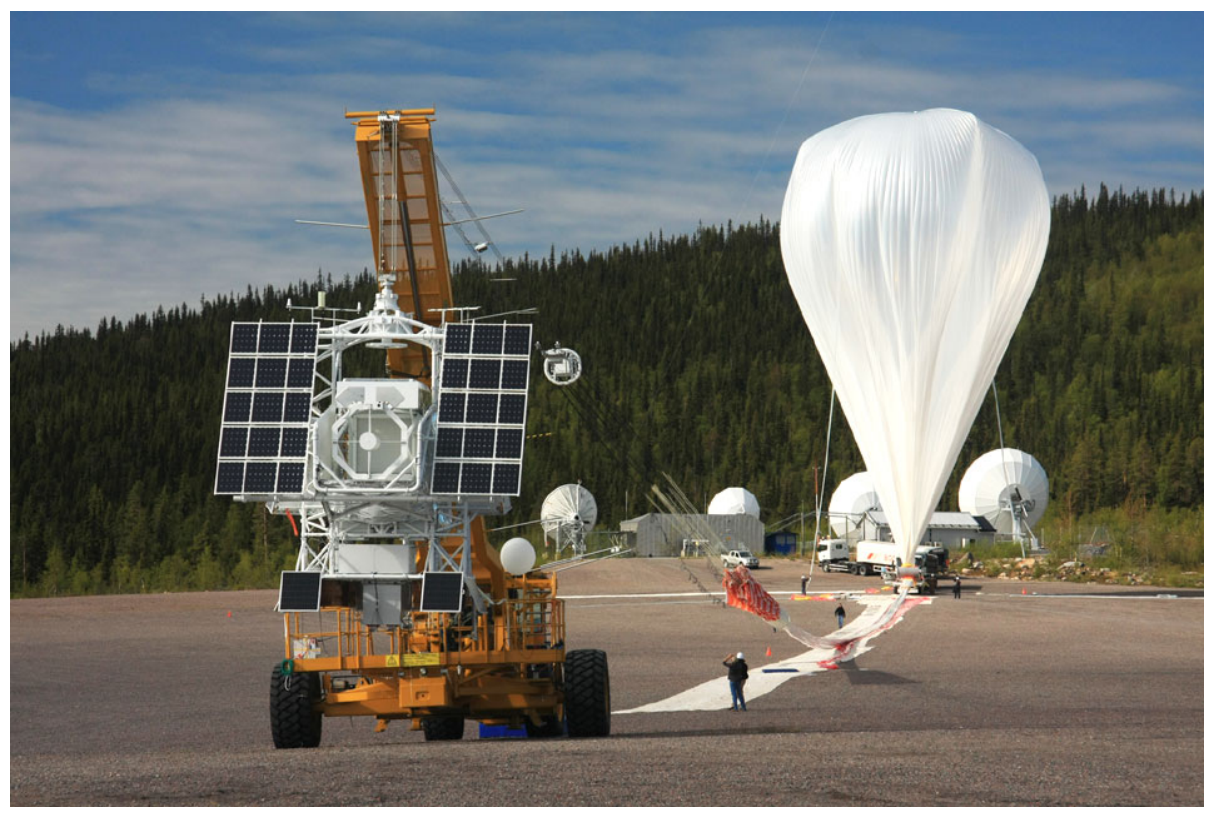

Figure 14 Sunrise immediately before launch. The balloon is only partly filled on ground to allow expansion with decreasing outside air pressure during ascent. At $37 \mathrm{~km}$ altitude it will have expanded by a factor of 300 as compared to ground. The parachute for landing is part of the flight train connecting the gondola payload with the balloon.

stability and stiffness, while minimizing weight. The NASTRAN finite element software package has been used. Several requirements had to be considered, such as instrument safety, alignment and pointing stability.

- Safety aspects are important when bringing a payload of several tons to stratospheric heights. CSBF requests to analytically prove instrument integrity with respect to mechanical loads induced at launch and flight termination. All components therefore have sufficient margin-of-safety to structural failure, for instance regarding vertical shock loads of up to $10 \mathrm{~g}$, expected during parachute opening.

- Mechanical loads induced during transport, ground handling and launch must not lead to permanent misalignment of structural components or instrument units. The design of all components and fixation devices considers a quasistatic load of $4 \mathrm{~g}$, leading to elastic deformations only.

- In contrast to a space instrument, Sunrise is subjected to gravity when in operation. The orientation of the telescope and instrumentation relative to the gravity vector varies significantly with the changing elevation of the Sun. This could affect the relative alignment of structural components or instruments. The telescope Serrurier structure as well as the PFI support structure provide sufficient structural stiffness to maintain optimal instrument performance. A few residual thermo-elastic deformations cannot be controlled purely by passive structural design. Therefore, the critical relative positioning of telescope primary and secondary mirror with a sensitivity in the micrometer range is actively adjusted in flight, ensuring the high performance image quality of the system.

- The servo control loops providing the gondola pointing assume rigid body motions when activating the azimuth and elevation drives. Rigid body motion is only given when the 
lowest eigenfrequency of the moving parts is considerably higher than the excitation frequencies. As the elevation and azimuth drives operate at frequencies below $10 \mathrm{~Hz}$, the gondola as largest structure has been designed to a minimum eigenfrequency of the order of $15 \mathrm{~Hz}$. Decoupling of eigenfrequencies avoids excitation of one subsystem by another. The PFI has been designed to a minimum eigenfrequency of $25 \mathrm{~Hz}$ and the telescope to $35 \mathrm{~Hz}$.

\subsubsection{Thermal Design}

Thermal analysis and thermal design is important to keep all instrument components within their specified temperature ranges, under all conditions. This is especially relevant for the high power dissipating commercial electronics and alignment-critical optics. The environmental conditions for Sunrise at stratospheric altitudes are very different compared to ground. Owing to the low pressure of only a few millibars, convective coupling is no longer the dominant heat exchange mechanism and the thermal behaviour of the instrument is controlled practically only by radiative heat exchange. Component temperatures result from the equilibrium of dissipated, absorbed, and emitted energy. Surface properties such as absorption and emission coefficients as well as view factors of surface elements with respect to their neighborhood and to heat sources and sinks are dominant factors.

During flight, Sunrise is exposed to a changing environment. The albedo and thus the heat input from below significantly varies when flying over sea, ice or cloud layers. In addition, the radiative input varies with solar elevation angle.

Two extreme steady state load cases covering the wide range of conditions to be expected were defined:

- A 'hot' case with 45-deg solar elevation angle, a solar flux of $1397 \mathrm{~W} \mathrm{~m}^{-2}$, an albedo of 0.95 simulating complete ice coverage, and Earth IR radiative input of $264 \mathrm{~W} \mathrm{~m}^{-2}$.

- A 'cold' case with 0-deg solar elevation angle, a reduced solar flux of $1044 \mathrm{~W} \mathrm{~m}^{-2}$, an albedo of 0.11 simulating sea water below the instrument, and Earth radiative input of $156 \mathrm{~W} \mathrm{~m}^{-2}$.

Sunrise with all components was modelled with several thousand nodes using the ESARAD/ESATAN software package. Geometries, surface properties and radiator sizes were determined and optimized.

Thermal Control of Instrument Electronics and Structural Components Heat dissipating elements such as computers, photovoltaic arrays etc. have been placed as far away as possible from the telescope and instruments. The instrument electronics are located on two aluminium honeycomb racks of $1 \times 2 \mathrm{~m}^{2}$ size, left and right on the rear side of the gondola. The racks are shaded by, and turned away from the solar arrays, minimizing the view factor of the electronics to the nearly $100^{\circ} \mathrm{C}$ hot photovoltaic cells. A $20^{\circ}$ inclination reduces albedo input and maximizes the view to the cold sky, while still keeping the moments of inertia of the system low. White paint is used as surface treatment for all the electronics units and passive structural components directly exposed to the Sun, such as the tubing of the gondola and the telescope mechanical components. Thermal filler is used underneath some of the electronics, enhancing the conductive coupling to the top surfaces of the racks so that they serve as additional radiating areas.

Gondola Blankets The impact of the changing environment on telescope and instrumentation must be minimized to reduce thermo-mechanical deformations. Shading solar radiation 
coming from the extended source below the observatory is provided by large thermal blankets, which are mounted to the gondola interior sides and bottom, including front and rear roll cage. The outer blanket layer is facing the Earth and reduces the heat input to the system. A Mylar foil of $125 \mu \mathrm{m}$ ( 5 mil) thickness with vapour deposited aluminium on one side was chosen. The foil is used as second surface mirror, providing similar thermal properties as white paint. The innermost layers of the gondola thermal blankets control the heat exchange with the telescope and science instrumentation. They are directly illuminated by the Sun during observation. Dunmore Beta Cloth $500 \mathrm{~F}$ is used in the front roll cage. This low-outgassing, space approved material is tailored to form a diffusely reflecting white cavity around the front end of the telescope, reducing the probability of structural hot spots. The interior of the rear roll cage is covered with a highly reflective aluminized polyimide (Kapton) foil, enhancing the radiative exchange of the primary mirror with the cold sky. Here the aluminium side is facing outwards, maximizing the reflection properties. Heating of the foil is only moderate, due to the grazing solar incidence on the roll cage sides and the shadowing by the telescope and instrumentation.

PFI Thermal Design The thermal design of the postfocus instrumentation places emphasis on thermal stability. The overall temperature level including gradients across the 2-m long structure have to be kept within a small temperature range of $20 \pm 10^{\circ} \mathrm{C}$. This ensures not only invariant alignment for non-carbon-fiber-based instruments as IMaX, but also minimizes changes of the polarization properties of the optics.

The PFI structure is decoupled from the environment by Styrofoam insulation, a low outgassing closed-cell material. Panels of 4-cm thickness are used on all four sides of the PFI, and 1-cm panels on the bottom towards the telescope. The panels are tailored to fit to the structure and wrapped and taped in aluminized Mylar foil of $125-\mu \mathrm{m}$ thickness, again used as second surface mirror. The foil wrapping minimizes particulate contamination of the optics, which could occur when using cut foam pieces. The top side of the PFI has a large view factor to the heat sink of the cold sky above the instrument. Structural components are covered with wrapped Styrofoam, similar to the PFI sides and bottom. White painted radiator plates serve as direct link for heat-dissipating proximity electronics, such as mechanism controllers, drive electronics for the tip/tilt mirror and SuFI camera head, electronics and power supply (Figure 9b). Fine adjustment of the expected electronics temperatures was achieved by partially covering the radiator areas with wrapped foam pieces. Groups of thermostat-controlled and actively controlled foil heaters were placed on the metallic covers of the ISLiD, SuFI, and CWS instruments underneath the foam insulation. A heat shield on the illuminated front end shades the PFI from direct solar radiation and minimizes temperature gradients in addition to the foam insulation.

Wind Shields The balloon ascent through the cold tropopause layer is considered as a risk for the PFI instrumentation and instrument electronics. Convective cooling in conjunction with the radiative heat exchange can lead to a critical temperature drop during this phase (see below). Dedicated wind shields were developed to reduce the impact of the cold air stream while not adversely affecting the thermal conditions at float. A $37.5 \mu \mathrm{m}$ (1.5 mil) Polyethylene film from the NASA ULDB program was selected and kindly provided by Aerostar Industries free of charge. This material is optimized in terms of mechanical durability at low temperature conditions and provides a high transparency in the visible and the infrared. The wind shield material and mounting concept was tested to withstand wind gusts and velocities up to $100 \mathrm{~km} \mathrm{~h}^{-1}$. 


\section{Mission}

\subsection{Subsystem Test Flights}

The gondola system and large parts of the instrument control system including the main computer (ICU), data storages and power distribution units, performed a $9 \mathrm{~h}$ test flight on 3 October 2007 from Ft. Sumner, New Mexico, USA. The gondola was equipped with a small UV telescope with $25 \mathrm{~cm}$ aperture and a camera system with a filter wheel, verifying the pointing capabilities of the gondola. Fully powered by batteries, the gondola carried solar panel dummies, simulating the thermal behaviour and being representative in terms of size, mass, inertia and aerodynamics. Pointing, onboard data acquisition, commanding and downlink through telemetry systems were tested. The payload was recovered largely intact on a wheat field close to Dalhart, Texas, USA, the following day.

The test flight revealed the sensitivity of some of the electronic equipment when exposed to the severe environmental conditions around the tropopause. Low temperatures of less than $-75^{\circ} \mathrm{C}$ (Pérez-Grande et al., 2009) caused the ICU to temporarily stop working. The ICU recovered at float altitude and performed flawlessly during nominal flight operation. For the science flight it was decided to modify the thermal design of the Sunrise instrument by implementing dedicated wind shields (see Section 2.7.2). A verification of this concept was performed on a dedicated stratospheric balloon test flight of electronics equipment in June 2008 from ESRANGE.

\subsection{Instrument Integration and Mission Preparations}

The two major components of Sunrise, the gondola and the telescope with its instrumentation package, have been assembled, integrated and tested separately at their respective home institutions, before being shipped to ESRANGE for system integration.

The gondola had survived the 2007 Ft. Sumner test flight relatively unharmed. The refurbishment was done at HAO. A few mechanical components needed replacement (crash pad assembly and roll cages) and some electrical modifications were implemented at HAO. Significant work was spent improving and testing the pointing control software.

At MPS the postfocus instrumentation (PFI) including ISLiD/SuFI was assembled and tested. The two externally provided, pre-aligned instrument units CWS and IMaX then were integrated and co-aligned relative to ISLiD/SuFI. Final verification on PFI level included checks of the overall wavefront quality. Polarization properties of the integrated package as well as the closed-loop performance of the tip/tilt mirror were measured.

The telescope was assembled, aligned and tested at Kayser-Threde, Munich, under contract by MPS. After delivery to MPS end of 2008, all relevant functional tests were repeated, including wavefront measurements with an interferometric end-to-end test and a verification of M2 movements to micrometer accuracy. The tests proved the excellent stability of the telescope system and verified the transportation concept with a dedicated damped telescope dolly tailored to fit into a standard sea container.

Telescope and postfocus instrumentation were mated for final instrument performance tests at MPS. Lasertracker measurements helped to align the PFI package on top of the central telescope frame to its nominal position. The four struts supporting the PFI edges needed careful adjustment to avoid any distortion or bending of the large structure, in order to reproduce the alignment status achieved earlier on the optical table.

Due to transport size limitations the PFI had to be dismounted from the telescope again for shipment. Both units were packed separately in vibration damped transport dollies and 
stowed in two $20 \mathrm{ft}$ sea containers. All equipment from HAO and MPS arrived end of March 2009 at the launch site ESRANGE.

The Sunrise flight hardware and auxiliary equipment were set up in the integration hangar nicknamed 'Cathedral' with approx. $250 \mathrm{~m}^{2}$ floor space. Functional tests of all units, alignment verification of the PFI and interferometric wavefront measurements on the telescope proved that all systems had survived the transport without degradations. PFI and telescope were mated on 11 April 2009, integration into the gondola was done one week later on 18 April 2009.

Extensive in-door testing was performed with the nearly fully assembled instrument, being suspended by the hangar crane. Parameters of the pointing system control loops were adjusted to the real moments of inertia of the flight hardware. A $10-\mathrm{kW}$ tungsten theater light was used as artificial sun. Although not giving the radiance levels comparable to direct sunlight, the response of all pointing relevant sensors such as corner cells, mid level sensors and the fine guider could be tested and the system response was optimized. Pointing tests were continued with real sunlight from within the 'Cathedral' the following days, further refining the control loop performance, now with higher photon flux on the sensors.

Sunrise instrument 'first light' was achieved on 30 April 2009. Tests verified the performance of the heat rejection wedge cooling, then first images were taken with the science instruments. Due to the very poor ground seeing conditions and the absence of sunspots no image stabilization was possible as no features were visible on the Sun. However, the exposure times for SuFI, IMaX and CWS could partly be verified in flatfield images, and the important wavelength calibration of IMaX could be performed.

Autonomous operation of the instruments needs predefined timelines of commanding. Those were extensively tested in May 2009, harmonizing and optimizing the various software codes of the different instrument units.

A full flight configuration compatibility test was successfully conducted on 30 May 2009 together with CSBF and ESRANGE. Sunrise was moved from the 'Cathedral' to the outside, all additional flight equipment was mounted. The test demonstrated electro-magnetic compatibility of all components and as well mechanical compatibility with regard to the launch procedures. Sunrise declared flight readiness on 5 June 2009, after having performed a last Sun-pointing test with IMaX calibrations.

\subsection{Flight June 2009}

Sunrise was successfully launched on 8 June 2009 at 6:27 UT (08:27 h local time), on its first launch opportunity. Balloon and instrument reached an initial float altitude of $37.2 \mathrm{~km}$ (122000 ft) after about three hours ascent.

Instrument commissioning started with the gondola pointing system. During ascent, Sunrise was in a rotary mode, providing solar illumination on virtually all parts of the instrument and thus some heating during the critical tropopause transit. This mode, however, required the whole system to be run on the limited battery power. Having adjusted relevant parameters of the pointing control loops to the different conditions due to the longer flight train, the telescope aperture door was opened at 11:41:15 UT. Instrument checkout and commissioning proceeded with the CWS, closing the loop for image stabilization and adjusting the focus of the telescope first at 13:20 UT. At that time, first images were taken by SuFI and IMaX. A full commissioning of the instruments was, however, impossible due to an early failure of the line-of-sight communication. The $2 \mathrm{Mbits}^{-1}$ high 


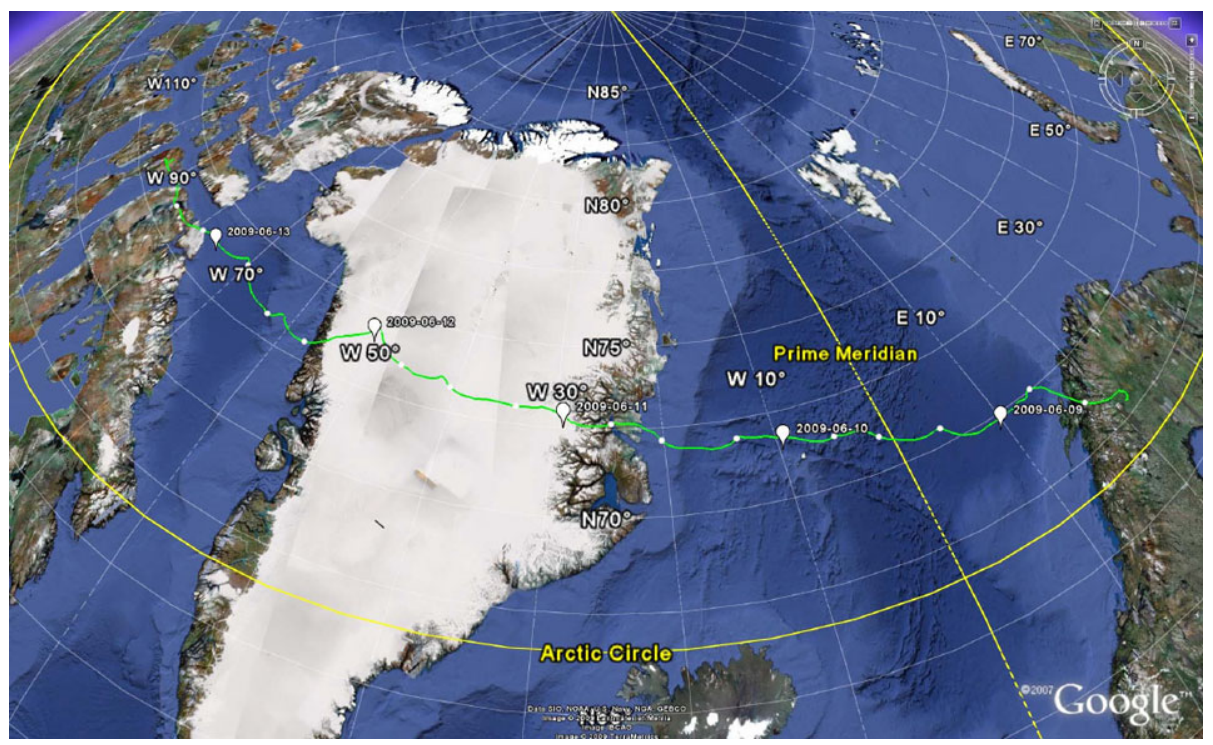

Figure 15 Flight trajectory of Sunrise.

speed telemetry link operated until 15:09 UT, but was unreliable towards the end. The handover to the second ground station in Andenes/Norway unfortunately failed. This additional ground station would have extended the communication range to approximately $24 \mathrm{~h}$ coverage, given the low speed and direction Sunrise was heading. The TDRSS link had to be used instead, providing a downlink data rate of approximately $6 \mathrm{kbits}^{-1}$. With this low data rate, some of the originally foreseen commissioning tasks could not be performed as planned.

The observation program collected minimum science data at disk center from 8 to 9 June 2009. On 10 June beginning at 01:36 UT co-alignment of telescope and sun sensor LISS was checked by searching the solar limb. Instrument flatfielding was performed by commanding a circular movement of the telescope pointing with fixed tip/tilt mirror.

Zonal winds carried the balloon and instrument with almost constant speed of $30 \mathrm{~km} \mathrm{~h}^{-1}$ to Northern Canada (Figure 15). The balloon altitude varied between $37 \mathrm{~km}$ and $34 \mathrm{~km}$, following the sun elevation with a phase lag of approximately 3 to $4 \mathrm{~h}$. Several ballast drops in the second half of the mission helped to regain float altitude.

Flight termination sequences were commanded on 13 June 2009 at 21:20 UT, followed by the balloon cut-away at 22:52 UT. Sunrise landed on Somerset Island, Nunavut County, Northern Canada at 23:44 UT after $137 \mathrm{~h}$ mission elapsed time and a travel distance of nearly $4350 \mathrm{~km}$.

Sunrise was completely recovered a few days after landing. All instrument parts were flown out via helicopter first to Resolute Bay, then with airplanes to Yellowknife, where the equipment was packed into sea containers for shipment to their home institutions. Damage to Sunrise was found to be moderate, e.g., the primary mirror survived the landing perfectly intact. The scientific data stored on the data storage harddrives safely arrived at MPS on 25 June 2009, being handcarried directly from Yellowknife on commercial airplanes. 


\section{Instrument In-Flight Performance}

The Sunrise instrument impressed with near flawless performance during its maiden flight, although instrument commissioning could not be fully accomplished due to the short period of line-of-sight high speed communication (see the previous section).

Power System, Electronics and Software All onboard electronics, as power system, instrument computers, proximity electronics and their related software, performed nominally during most of the mission.

The sizing of battery capacity and solar panels proved to be very conservative. Although the time to first Sun acquisition was about $2 \mathrm{~h}$ longer than anticipated (see below), the battery charge state did not drop below $77 \%$. Once correctly oriented, the solar panels fully recharged the batteries within $10 \mathrm{~h}$. The system current plus the charge current to the batteries were provided by only three - four out of the ten panels. The charge controller automatically deactivated the unused panels.

Some issues were identified concerning the handling of the data storage units. On the second day (9 June) the instrument control unit had to be rebooted. It was unable to recover from a failed write operation to one of the active disks. During the data post-processing, it became obvious that the disk showed signs of malfunction already before that, since there were files missing from the observations made during the first night. However, all data were successfully recovered due to the applied RAID functionality.

Thermal All component temperatures remained well within their operational design limits and close to their predictions given by the detailed thermal models.

During ascent all components covered by wind shields - PFI and electronics racks - encountered only minimal temperature drops to about $0^{\circ} \mathrm{C}$, when passing the cold tropopause layer. Temperatures of unshielded components, as the gondola structure, the solar panels or the telescope trusses, dropped to about $-40^{\circ} \mathrm{C}$ at the same time. At float, the temperatures for instrument computers mounted to the electronics racks stayed within $0^{\circ} \mathrm{C}$ and $+30^{\circ} \mathrm{C}$ (see Pérez-Grande et al., 2010; Berkefeld et al., 2010). Similar temperatures were measured for the proximity electronics, as mechanism controllers within the PFI structure. The optical components and mechanisms inside the PFI instrumentation showed temperatures between $+5^{\circ} \mathrm{C}$ and $+25^{\circ} \mathrm{C}$ with a diurnal variation of only $\approx 8^{\circ} \mathrm{C}$, thus perfectly maintaining the alignment as adjusted in the laboratory. Several external components showed temperature variations of typically $\pm 10^{\circ} \mathrm{C}$. They are mainly caused by the changing albedo flux onto the instrument surfaces, resulting from changing solar elevation and terrain underneath the gondola.

The varying albedo flux also slightly influenced telescope component temperatures, although the telescope remained nearly permanently oriented towards the Sun. Figure 16 shows a plot of three temperature sensors mounted to the Zerodur primary mirror at various lateral positions as well as the temperature measured at the field stop in the primary focal plane (heat rejection wedge). The large mirror showed only negligible temperature gradients across the aperture of less than $10^{\circ} \mathrm{C}$. More important, both mirror and heat rejection wedge had very moderate temperatures below $+22^{\circ} \mathrm{C}$ throughout the flight, less than $30^{\circ} \mathrm{C}$ above the free air temperature at float altitude, which was measured to be around $-6^{\circ} \mathrm{C}$. This temperature difference is low enough to exclude the risk of wavefront aberrations due to 'mirror seeing'. 
Figure 16 In-flight temperatures of three primary mirror sensors at several positions distributed over the aperture and of the heat rejection wedge at the primary focus (in red).

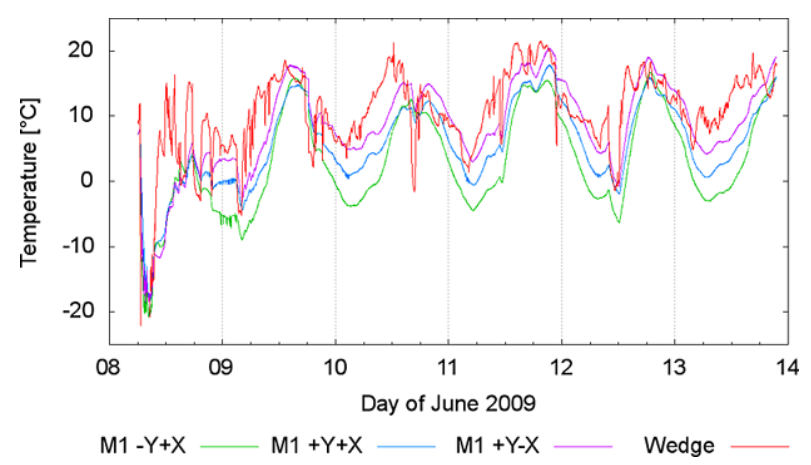

Wavefront Quality The optical performance of the instrument can be derived from data of the CWS instrument, directly measuring the wavefront quality in flight, as well as from the phase diversity image analysis and reconstruction. Both data sources prove the excellent end-to end performance and stability of the telescope and postfocus instrumentation.

The diurnal thermo-elastic deformations and the varying orientation of the optical system with respect to the gravity vector lead to small variations in the relative positioning of the primary and secondary telescope mirrors. Resulting aberrations, as defocus and coma, are measured by the wavefront sensor CWS. They can be corrected by secondary mirror displacements. During flight only the focus was adjusted in closed-loop by continuous axial repositioning of $\mathrm{M} 2$ in a range of about $100 \mu \mathrm{m}$. Uncorrected, this defocussing corresponds to a wavefront error of $\pm 0.5 \lambda \mathrm{rms}$. The CWS sensitivity and the micrometer accuracy of the M2 translation stage helped to achieve focus accuracies below $0.01 \lambda$ rms (Berkefeld et al., 2010). Lateral displacements of M2 would have caused a significant image displacement, deteriorating the co-alignment of telescope and LISS sun sensor. The observed uncorrected coma values were less than $\lambda / 10$, small enough to be handled by the phase diversity algorithms. An additional in-flight coma correction was therefore not performed.

Analysis of the SuFI images shows a total wavefront error in the order of $\lambda / 5$ to $\lambda / 6$ rms at $300 \mathrm{~nm}$, very close to the expected performance (see Gandorfer et al., 2010). Main contributors are astigmatism and trefoil, most probably originating from the primary mirror, showing some dependance on elevation angle (Hirzberger et al., 2010a). Similar results were obtained from phase diversity analysis of IMaX data (Martínez Pillet et al., 2010). Differential focus between CWS and SuFI as well as CWS and IMaX was found to be within $\lambda / 20$.

Pointing Performance The gondola pointing system worked very reliably, keeping the instrument autonomously oriented towards the Sun. The first Sun acquisition with aperture door opening occurred 5:15 h after launch, about $2 \mathrm{~h}$ later than expected. The tropopause transit had caused a temporary freeze of the fine azimuth motor, which unfortunately was not switched to rotary mode during ascent as planned for continuous heating. Once this system was recovered, part of the commissioning time with high speed telemetry was used to adapt gain and filter settings, optimizing the system response on the long flight train.

The wind gusts acting on the gondola were unfortunately strong enough to reduce the pointing performance quite frequently. Flight data indicate that performance was best over the free ocean between Norway and Greenland (9 June) and less good over Greenland and the many coast lines of Northern Canada (13 June). Higher float altitudes in general seem to be beneficial for the pointing stability, due to the lower air density. The longest gondola pointing within the \pm 46 arcsec capture range of the image stabilization system was $45 \mathrm{~min}$. 
Forty-seven time series with durations between 10 and 45 min were obtained with closedloop image stabilization (see Berkefeld et al., 2010). In total more than $33 \mathrm{~h}$ of science observation (23\% of the total observing time) were obtained. Details on SuFI and IMaX observing modes, number of acquired images and duration of time series are given in Solanki et al. (2010). The telescope aperture door was closed 93 times due to pointing errors exceeding 15 arcmin. No indication of structural damage to the telescope components in the vicinity of the primary focus was found.

Post-flight analysis of the image data gives evidence that the imaging performance of Sunrise is not limited by the wavefront error of the telescope and instrument optics, but by a residual image smear of the order of 0.03-0.04 arcsec (Berkefeld et al., 2010), which reduces the spatial resolution in case of SuFI to $\approx 0.1 \operatorname{arcsec}$ (Gandorfer et al., 2010) and to 0.15-0.18 arcsec for IMaX (Martínez Pillet et al., 2010).

Although the CWS system was able to dramatically improve the effective pointing stability, not all components of the incoming excitation spectrum of the balloon/gondola system could be damped out.

Figure 17 shows LISS Sun sensor data obtained on 9 June 2009 with $150 \mathrm{~Hz}$ sampling rate. The sample is a representative part of $\mathrm{a} \approx 40 \mathrm{~min}$ period, when the gondola pointing remained within the \pm 46 arcsec capture range of the tip/tilt mirror and both image stabilization and telescope focus loops were closed. The data clearly show a slight elevation pendulum motion (pitch), as well as an azimuthal (yaw) oscillation with a frequency of $10 \mathrm{~Hz}$ and several arcsec amplitude. This oscillation most likely can be attributed to an excited resonance in the mechanical structure of the gondola (bending mode of the solar panels), influencing the pointing system. Amplitudes varied over the mission and increased by up to a factor of three towards the end.
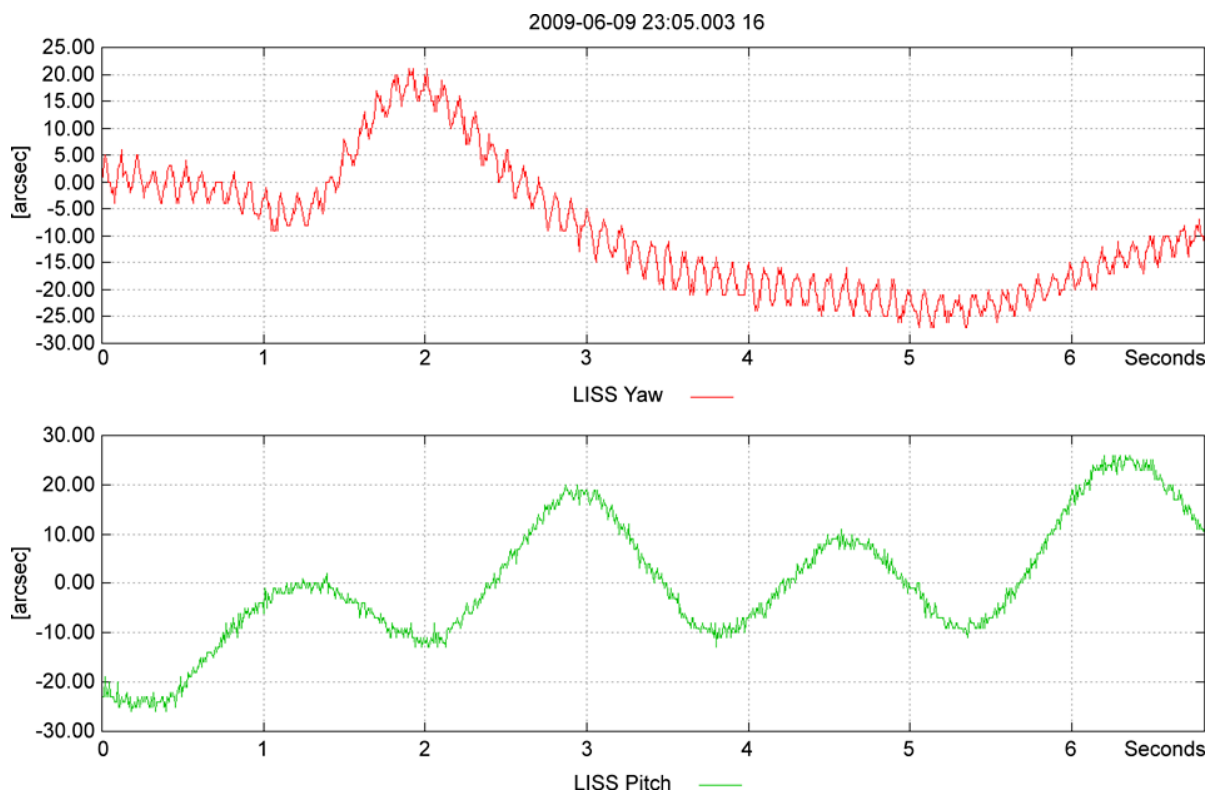

Figure 17 LISS Sun Sensor data (in arcsec) sampled with $150 \mathrm{~Hz}$ on 9 June 2009, 23:02:05 UT. The length of the interval shown is $6.8 \mathrm{~s}$, being a representative part of $\mathrm{a} \approx 40 \mathrm{~min}$ period of closed loop pointing. A $10 \mathrm{~Hz}$ oscillation in azimuth (yaw) can clearly be seen, as well as slight pendulation in elevation (pitch). 
A Fourier analysis of residual image stabilization data taken on 12 June (see Berkefeld et al., 2010, Figure 18) clearly shows this $10 \mathrm{~Hz}$ peak in the power spectra, but also reveals additional components at frequencies above $40 \mathrm{~Hz}$, where the image stabilization is already quite limited. Both contributions immediately died out when the azimuth motors were switched off. This can be documented by accelerometer data taken at several positions on the gondola structure, showing higher amplitudes at the gondola top. We therefore currently speculate that the excitation of the $10 \mathrm{~Hz}$ oscillation and high frequency jitter is connected with the azimuth drives, e.g. by bearing rumble. A detailed analysis of these phenomena is still ongoing, also regarding the relative contribution to the observed image smear.

\section{Outlook}

During its 137-h successful flight Sunrise collected a unique data set. The payload remained in direct sunlight during the entire flight. Seeing-free observations were possible all the time. The total observing time with image stabilization was more than $33 \mathrm{~h}$, in which SuFI collected nearly 56000 images in the wavelength range between $210 \mathrm{~nm}$ to $400 \mathrm{~nm}$, being above most of the ozone layer in the Earth's atmosphere. In the blue channels at $214 \mathrm{~nm}, 300 \mathrm{~nm}$, and $312 \mathrm{~nm}$, the solar surface could be observed for the first time at high angular resolution and with unprecedented intensity contrasts (Solanki et al., 2010; Riethmüller et al., 2010; Hirzberger et al., 2010b), thanks to the high optical quality of the instrumentation. IMaX collected high resolution Doppler- and magnetograms with an unprecedented combination of angular resolution and magnetic sensitivity (Martínez Pillet et al., 2010).

The Sun was extremely quiet during the 2009 science flight. Although the observation of quiet solar granulation is in itself an important scientific aspect of Sunrise, a more complete sampling of the different aspects of solar surface magnetism is needed to finally help us understanding the underlying physical roots of solar activity. Since the Sunrise instrumentation showed an excellent performance during flight and survived the landing and recovery with only minor damage, a reflight of Sunrise in phases of higher solar activity has the potential of significantly advancing our knowledge of the Sun, overcoming the current performance limitations with relatively low additional effort.

Acknowledgements The German contribution to Sunrise is funded by the Bundesministerium für Wirtschaft und Technologie through Deutsches Zentrum für Luft- und Raumfahrt e.V. (DLR), Grant No. 50 OU 0401, and by the Innovationsfond of the President of the Max Planck Society (MPG). The Spanish contribution has been funded by the Spanish MICINN under projects ESP2006-13030-C06 and AYA2009-14105-C06 (including European FEDER funds). HAO/NCAR is sponsored by the National Science Foundation. The HAO contribution was partly funded through NASA grant number NNX08AH38G. We greatly appreciate the tremendous support provided by CSBF for this mission, especially by Danny Ball. We also would like to thank all current and previous team members not listed as co-authors for their valuable contribution to the project.

The quality of the manuscript was greatly enhanced during the review process. We would like to thank the anonymous referee for her/his insightful and helpful comments.

Open Access This article is distributed under the terms of the Creative Commons Attribution Noncommercial License which permits any noncommercial use, distribution, and reproduction in any medium, provided the original author(s) and source are credited.

\section{References}

Bernasconi, P.N., Rust, D.M., Eaton, H.A., Murphy, G.A.: 2000, In: Melugin, R.K., Roeser, H.P. (eds.) Airborne Telescope Systems, Proc. SPIE 4014, 214. 
Bernasconi, P.N., Eaton, H.A., Foukal, P., Rust, D.M.: 2004, Adv. Space Res. 33, 1746.

Berkefeld, T., Schmidt, W., Soltau, D., Bell, A., Doerr, H.P., Feger, B., et al.: 2010, Solar Phys. doi:10.1007/ s11207-010-9676-3.

Danielson, R.E.: 1961, Astrophys. J. 134, 275.

Gandorfer, A., Grauf, B., Barthol, P., Riethmüller, T.L., Solanki, S.K., Chares, B., et al.: 2010, Solar Phys. doi:10.1007/s11207-010-9636-y.

Herse, M.: 1979, Solar Phys. 63, 35.

Hirayama, T.: 1978, Publ. Astron. Soc. Japan. 30, 337.

Hirzberger, J., Feller, A., Riethmüller, T.L., Gandorfer, A., Solanki, S.K.: 2010a, Astron. Astrophys. doi:10.1088/2041-8205/723/2/L154.

Hirzberger, J., Feller, A., Riethmüller, T.L., Schüssler, M., Borrero, J.M., Afram, N., et al.: 2010b, Astrophys. J. Lett., in press.

Krat, V.A., Karpinsky, V.N., Pravdjuk, L.M.: 1972, Solar Phys. 26, 305.

Lemaire, P., Samain, D.: 1984, Adv. Space Res. 4, 37.

Lemaire, P., Blamont, J.E.: 1967, Astrophys. J. Lett. 150, 129.

Martínez Pillet, V., del Toro Iniesta, J.C., Álvarez Herrero, A., Domingo, V., Bonet, J.A., González Fernández, L., et al.: 2010, Solar Phys. doi:10.1007/s11207-010-9644-y.

Mehltretter, J.P.: 1978, Astron. Astrophys. 62, 311.

Pérez-Grande, I., Sanz-Andrés, A., Bezdenejnykh, N., Barthol, P.: 2009, Appl. Thermal Eng. 29, 1507.

Pérez-Grande, I., Sanz-Andrés, A., Bezdenejnykh, N., Farrahi, A., Barthol, P., Meller, R.: 2010, J. Aero. Eng. Part $C$, submitted.

Riethmüller, T.L., Solanki, S.K., Martínez Pillet, V., Hirzberger, J., Feller, A., Bonet, J.A., et al.: 2010, Astrophys. J. Lett. doi:10.1088/2041-8205/723/2/L169.

Rust, D.M., Murphy, G., Strohbehn, K., Keller, C.U.: 1996, Solar Phys. 164, 403.

Schwarzschild, M.: 1959, Astrophys. J. 130, 345.

Solanki, S.K., Feller, A., Gandorfer, A., Hirzberger, J., Riethmüller, T.L. Schüssler, et al.: 2010, Astrophys. J. Lett. doi:10.1088/2041-8205/723/2/L127. 